\title{
Finite-Temperature Form Factors: a Review ${ }^{\star}$
}

\author{
Benjamin DOYON
}

Rudolf Peierls Centre for Theoretical Physics, Oxford University, 1 Keble Road, Oxford OX1 3NP, U.K.

E-mail: b.doyon1@physics.ox.ac.uk

URL: www-thphys.physics.ox.ac.uk/user/BenjaminDoyon

Received October 09, 2006, in final form December 07, 2006; Published online January 11, 2007

Original article is available at http://www.emis.de/journals/SIGMA/2007/011/

\begin{abstract}
We review the concept of finite-temperature form factor that was introduced recently by the author in the context of the Majorana theory. Finite-temperature form factors can be used to obtain spectral decompositions of finite-temperature correlation functions in a way that mimics the form-factor expansion of the zero temperature case. We develop the concept in the general factorised scattering set-up of integrable quantum field theory, list certain expected properties and present the full construction in the case of the massive Majorana theory, including how it can be applied to the calculation of correlation functions in the quantum Ising model. In particular, we include the "twisted construction", which was not developed before and which is essential for the application to the quantum Ising model.
\end{abstract}

Key words: finite temperature; integrable quantum field theory; form factors; Ising model

2000 Mathematics Subject Classification: 81T40

\section{Introduction}

Relativistic quantum field theory (QFT) at finite temperature is a subject of great interest which has been studied from many viewpoints (see, for instance, [1]). An important task when studying a model of QFT is the calculation of correlation functions of local fields, which are related to local observables of the underlying physical model. For instance, two-point correlation functions are related to response functions, which can be measured and which provide precise information about the dynamics of the physical system at thermodynamic equilibrium. Although applications to particle physics often can be taken to be at zero temperature, many applications to condensed matter require the knowledge of the effect of a non-zero temperature on correlation functions.

In this article, we review and develop further the ideas of [2] for studying finite-temperature correlation functions in integrable quantum field theory.

In recent years, thanks to advances in experimental techniques allowing the identification and study of quasi-one-dimensional systems (see for instance [3, 4]), there has been an increased interest in calculating correlation functions in 1+1-dimensional integrable models of QFT (for applications of integrable models to condensed matter systems, see for instance the recent review [5]). Integrable models are of particular interest, because in many cases, the spectrum of the Hamiltonian in the quantization on the line is known exactly (that is, the set of particle types and their masses), and most importantly, matrix elements of local fields in eigenstates of the Hamiltonian, or form factors, can be evaluated exactly by solving an appropriate RiemannHilbert problem in the rapidity space $[6,7,8,9,10]$.

${ }^{\star}$ This paper is a contribution to the Proceedings of the O'Raifeartaigh Symposium on Non-Perturbative and Symmetry Methods in Field Theory (June 22-24, 2006, Budapest, Hungary). The full collection is available at http://www.emis.de/journals/SIGMA/LOR2006.html 
At zero temperature, correlation functions are vacuum expectation values in the Hilbert space of quantization on the line. The knowledge of the spectrum on the line and the matrix elements of local fields then provides a useful expansion of two-point functions at space-like distances, using the resolution of the identity in terms of a basis of common eigenstates of the momentum operator and of the Hamiltonian. This is a useful representation because it is a large-distance expansion, which is hardly accessible by perturbation theory, and which is often the region of interest in condensed matter applications. Form factor expansions in integrable models at zero temperature have proven to provide a good numerical accuracy for evaluating correlation functions in a wide range of energies, and combined with conformal perturbation theory give correlation functions at all energy scales (an early work on this is [11]).

One would like to have such an efficient method for correlation functions at finite (non-zero) temperature as well. Two natural (mathematically sound) ways present themselves:

- "Form factor" expansion in the quantization on the circle. It is a general result of QFT at finite temperature $[12,13,14]$ that correlation functions, at space-like distances, can be evaluated by calculating correlation functions of the same model in space-time with Euclidean (flat) metric and with the geometry of a cylinder, the "imaginary time" wrapping around the cylinder whose circumference is the inverse temperature. In this picture, one can quantize on the circle (that is, taking space as being the circle, and Euclidean time the line), and correlation functions become vacuum expectation values in the Hilbert space of this quantization scheme. Then, one can insert a resolution of the identity in terms of a complete set of states that diagonalise both the generator of time translations and of space translations, as before, and one obtains a large-distance expansion for finite-temperature correlation functions.

Unfortunately, the two ingredients required (the energy levels in the quantization on the circle and the matrix elements of local fields) are not known in general in integrable quantum field theory. We should mention, though, that exact methods exist to obtain nonlinear integral equations that define the energy levels (from thermodynamic Bethe ansatz techniques, from calculations à la Destri-de Vega and from the so-called BLZ program), and that matrix elements of local fields were studied, for instance, in [15, 16, 17, 18]. Also, in the Majorana theory, the spectrum is known (since this is a free theory), and matrix elements of the primary "interacting" twist fields were calculated in $[19,20]$ from the lattice Ising model, and in a simpler way in [21] directly in the Majorana theory using the free-fermion equations of motion and the "doubling trick".

- Spectral decomposition on the space of "finite-temperature states". The concept of finitetemperature states, interpreted as particle and hole excitations above a "thermal vacuum", was initially proposed more than thirty years ago and developed into a mature theory under the name of thermo-field dynamics [22, 23, 24] (for a review, see for instance [25]). Ideas of 1+1-dimensional integrable quantum field theory were not applied to this theory until recently. In [26], the concept of bosonization in thermo-field dynamics was studied, and, of most interest to the present review, in [2] the concept of finite-temperature form factor was developed - matrix elements of local fields on the finite-temperature Hilbert space. There, it was studied in depth in the free Majorana theory, both for general free fields (finite normal-ordered products of the free Majorana fermion fields - including the energy field) and for twist fields. It was found that a Riemann-Hilbert problem again characterises finite-temperature form factors of twist fields, but that this Riemann-Hilbert problem presents important modifications with respect to the zero-temperature case. Solutions were written explicitly for primary "order" and "disorder" twist fields, and the full finitetemperature form factor expansions of two-point functions were written and interpreted as Fredholm determinants. 
An interesting discovery of [2] is that these two methods are actually related: it is possible to evaluate form factors on the circle from (analytical continuations of) the finite-temperature form factors, and the analytical structure of finite-temperature form factors (and of the measure involved in the expansion of correlation functions) is directly related to the spectrum in the quantization on the circle. This provided a new way of evaluating form factors of twist fields on the circle, and most importantly, gave a clear prescription for the integration contours in the finite-temperature form factor expansion (naively plagued with singularities). The requirements brought on finite-temperature form factors by this relation constitute, in a way, a generalisation of the modularity requirements found in conformal field theory for constructing correlation functions from conformal blocks.

It is important to realise, though, that both expansions for correlation functions are not equivalent. The first one gives an expansion at large (space-like) distances, whereas the second can be used to obtain both large-distance and, expectedly with more work, large-time expansions. Indeed, the finite-temperature form factor expansion can naturally be deformed into an expansion in the quantization on the circle through the relation mentioned above [2]. It is expected that it can also be manipulated to obtain large-time behaviours. A manipulation of this type was done in [27]. There, going in reverse direction as what is described in [2], the expansion on the circle in the quantum Ising model was first deformed into a kind of finite-temperature form factor expansion (without being recognised as such), which was then used to obtain large-time dynamical correlation functions in a certain "semi-classical" regime (partly reproducing earlier results of [28] and [29]). This manipulation, however, neglected contributions that may change the asymptotic behaviour, and a more complete derivation of the large-time behaviours from finite-temperature form factor expansions is still missing. In particular, for the quantum Ising model, the Fredholm determinant representation of [2] and those obtained in the present paper may be of use, following the technology reviewed in [30] (work is in progress [31]).

It is worth noting that the method we review here is not adapted to providing information about one-point functions at finite-temperature. Various works exist concerning such objects $[32,33,34]$. Work [32] is interesting in that it uses the knowledge of the zero-temperature form factors in order to deduce the finite-temperature one-point function of the energy field. The idea is to "perform" directly the finite-temperature trace from the known matrix elements. A regularisation is necessary, but the finite-volume one seems impossible to tackle. A certain convenient regularisation was proposed there and shown to reproduce the known finite-temperature average energy. The idea of using this regularisation for multi-point correlation functions has been suggested and we are aware of results in this direction [35], but it is not yet understood why in general this should work.

Let us also mention that correlation functions of twist fields in the Majorana theory can be obtained as appropriate solutions to non-linear differential equations [36]. But at finite temperature, or on the geometry of the cylinder, these equations are partial differential equations in the coordinates on the cylinder [37, 38, 39], and do not immediately offer a very useful tool for numerically evaluating correlation functions, neither for analyzing their large-distance and large-time behaviours.

The theory developed in [2] for the Majorana case is still incomplete. Twist fields present certain complexifications at finite temperature that are not present at zero temperature, and, in order to describe all correlation functions, one also needs a "twisting" of the construction of [2], as it was mentioned there. In addition, certain exponential pre-factors were omitted in [2]. These two aspects are in fact essential for applications of the results in the Majorana theory to correlation functions in the quantum Ising model.

In this article we will review the ideas of [2], by developing them in the general factorised scattering context of integrable quantum field theory, and complete the work for the Majorana 
theory. We will deduce many of the immediate properties that arise in the general context for finite-temperature form factors, drawing on the ideas of [2], and we will present both the untwisted and the twisted constructions. We will recall the results for the Majorana theory, and extend them to the twisted case, finally giving the explicit representation for correlation functions in the quantum Ising model.

The article is organised as follows. In Section 2 we review the form factor program at zero temperature, and in Section 3 we recall basic results about finite-temperature correlation functions. Then, in Section 4, we describe the concept of finite-temperature states using the language of factorised scattering in integrable QFT, we introduce the concept of finite-temperature form factor and we describe the resulting expansion of correlation function. We also present the ideas underlying the relation between finite-temperature form factors and matrix elements in the quantization on the circle, still in the general context. In Section 5, we develop the basics of the twisted construction. In Section 6, we present certain formal results about the space of finite-temperature states, and in particular, we deduce a generalisation of the idea of "mapping to the cylinder" that one uses in conformal field theory in order to study correlation functions at finite temperature (again, this is a generalisation of ideas of [2]). Finally, in Section 7, we recall and extend the results of [2] for the Majorana theory and its connection to the quantum Ising model.

\section{Review of the zero-temperature form factor program in integrable quantum field theory}

The Hilbert space of massive relativistic quantum field theory is completely specified by fixing the set $E$ of particle types of the model. In $1+1$ dimensions, every Hamiltonian eigenstate is then described by choosing $k \in \mathbb{N}$ particle types and by associating to them $k$ real numbers, the rapidities:

$$
\left|\theta_{1}, \ldots, \theta_{k}\right\rangle_{a_{1}, \ldots, a_{k}}
$$

with $a_{i} \in E$ and $\theta_{i} \in \mathbb{R}$ (and the order of the rapidities/particle types is irrelevant - hence a basis is obtained by fixing an ordering of the rapidities). The Hamiltonian $H$ and the momentum $P$ act diagonally on these states. In order to fix their eigenvalues, one only has to fix the masses $m_{a} \in \mathbb{R}^{+}$for every particle type $a \in E$. The eigenvalues are then

$$
H: E_{k}=\sum_{i=1}^{k} m_{a_{i}} \cosh \theta_{i}, \quad P: p_{k}=\sum_{i=1}^{k} m_{a_{i}} \sinh \theta_{i} .
$$

Other symmetries of the model also act diagonally, and their eigenvalues are fixed by choosing charges associated to the various particle types.

There are many possible bases of the Hilbert space, all described as above. Two are of particular importance: the in basis and the out basis. They describe, respectively, particles of the given types and rapidities far in the past, and far in the future (in non-integrable models, one should really include the additional dependence on the impact parameters). The far past and the far future are regions in time where all particles are so far apart that they do not interact, and can be described as freely propagating. The overlap between the in basis and the out basis gives the scattering matrix:

$$
\left|\theta_{1}, \theta_{2}, \ldots\right\rangle_{a_{1}, a_{2}, \ldots}^{(i n)}=\sum_{a_{1}^{\prime}, a_{2}^{\prime}, \ldots} \int d \theta_{1}^{\prime} d \theta_{2}^{\prime} \ldots S_{a_{1}, a_{2}, \ldots}^{a_{1}^{\prime}, a_{2}^{\prime}, \ldots}\left(\theta_{1}, \theta_{2}, \ldots ; \theta_{1}^{\prime}, \theta_{2}^{\prime}, \ldots\right)\left|\theta_{1}^{\prime}, \theta_{2}^{\prime}, \ldots\right\rangle_{a_{1}^{\prime}, a_{2}^{\prime}, \ldots}^{(o u t)}
$$


where the number of particles in the in state and in the out states is generically different. The structure of the Hilbert space and the Hamiltonian describe the particles and their propagation, but it is the scattering matrix that encodes the interaction, and in particular, the locality of relativistic quantum field theory.

In integrable quantum field theory, the scattering matrix can be determined from the physical requirements of unitarity and crossing symmetry, from the integrability requirement of factorisation and the lack of particle production, and from minimality assumptions and the "nuclear democracy" (every pole has a physical explanation through resonances from particles already in the spectrum). All scattering processes can then be described using only the two-particle scattering matrix $S_{a_{1}, a_{2}}^{b_{1}, b_{2}}\left(\theta_{1}-\theta_{2}\right), \theta_{1}>\theta_{2}$ :

$$
\left|\theta_{1}, \theta_{2}\right\rangle_{a_{1}, a_{2}}^{(i n)}=\sum_{b_{1}, b_{2}} S_{a_{1}, a_{2}}^{b_{1}, b_{2}}\left(\theta_{1}-\theta_{2}\right)\left|\theta_{1}, \theta_{2}\right\rangle_{b_{1}, b_{2}}^{(\text {out })}
$$

It is convenient for this purpose to introduce the Zamolodchikov-Faddeev algebra (from now on in this section, summation over repeated indices will be implied)

$$
\begin{aligned}
& Z^{a_{1}}\left(\theta_{1}\right) Z^{a_{2}}\left(\theta_{2}\right)-S_{b_{1}, b_{2}}^{a_{1}, a_{2}}\left(\theta_{1}-\theta_{2}\right) Z^{b_{2}}\left(\theta_{2}\right) Z^{b_{1}}\left(\theta_{1}\right)=0, \\
& \bar{Z}_{a_{1}}\left(\theta_{1}\right) \bar{Z}_{a_{2}}\left(\theta_{2}\right)-S_{a_{1}, a_{2}}^{b_{1}, b_{2}}\left(\theta_{1}-\theta_{2}\right) \bar{Z}_{b_{2}}\left(\theta_{2}\right) \bar{Z}_{b_{1}}\left(\theta_{1}\right)=0, \\
& Z^{a_{1}}\left(\theta_{1}\right) \bar{Z}_{a_{2}}\left(\theta_{2}\right)-S_{a_{2}, b_{1}}^{b_{2}, a_{1}}\left(\theta_{2}-\theta_{1}\right) \bar{Z}_{b_{2}}\left(\theta_{2}\right) Z^{b_{1}}\left(\theta_{1}\right)=\delta_{a_{2}}^{a_{1}} \delta\left(\theta_{1}-\theta_{2}\right) .
\end{aligned}
$$

The in basis and the out basis are then two bases for the same Fock space (actually, a generalisation of the concept of Fock space) over this algebra, defined simply by different ordering of the rapidities:

$$
\begin{array}{ll}
Z^{a}(\theta)|\operatorname{vac}\rangle=0, & \\
\left|\theta_{1}, \ldots, \theta_{k}\right\rangle_{a_{1}, \ldots, a_{k}}^{(\text {in })}=\bar{Z}_{a_{1}}\left(\theta_{1}\right) \cdots \bar{Z}_{a_{k}}\left(\theta_{k}\right)|\operatorname{vac}\rangle & \left(\theta_{1}>\cdots>\theta_{k}\right), \\
\left|\theta_{1}, \ldots, \theta_{k}\right\rangle_{a_{1}, \ldots, a_{k}}^{(\text {out })}=\bar{Z}_{a_{1}}\left(\theta_{1}\right) \cdots \bar{Z}_{a_{k}}\left(\theta_{k}\right)|\operatorname{vac}\rangle & \left(\theta_{1}<\cdots<\theta_{k}\right) .
\end{array}
$$

The natural Hermitian structure on this space gives $\left(Z^{a}(\theta)\right)^{\dagger}=\bar{Z}_{a}(\theta)$.

Once the Hilbert space has been identified with the Fock space over the ZamolodchikovFaddeev algebra, the algebra elements $Z^{a}(\theta)$ and $\bar{Z}_{a}(\theta)$ become operators with an action on the Hilbert space. It turns out, from expected properties of quantum field theory, that they induce very nice properties on the objects (form factors)

$$
F_{a_{1}, \ldots, a_{k}}^{\mathcal{O}}\left(\theta_{1}, \ldots, \theta_{k}\right) \equiv\left\langle\operatorname{vac}\left|\mathcal{O}(0,0) \bar{Z}_{a_{1}}\left(\theta_{1}\right) \cdots \bar{Z}_{a_{k}}\left(\theta_{k}\right)\right| \operatorname{vac}\right\rangle,
$$

where $\mathcal{O}(x, t)$ is a local field of the model. Indeed, these objects, defined here for real rapidities, actually are (by analytical continuation) meromorphic functions of the rapidities. They can be determined through a set of analyticity requirements and through the recursive determination of the residues at the poles (form factor equations) [8, 10]:

1. Meromorphicity: as functions of the variable $\theta_{i}-\theta_{j}$, for any $i, j \in\{1, \ldots, k\}$, they are analytic inside $0<\operatorname{Im}\left(\theta_{i}-\theta_{j}\right)<2 \pi$ except for simple poles;

2. Relativistic invariance:

$$
F_{a_{1}, \ldots, a_{k}}^{\mathcal{O}}\left(\theta_{1}+\beta, \ldots, \theta_{k}+\beta\right)=e^{s(\mathcal{O}) \beta} F_{a_{1}, \ldots, a_{k}}^{\mathcal{O}}\left(\theta_{1}, \ldots, \theta_{k}\right),
$$

where $s(\mathcal{O})$ is the spin of $\mathcal{O}$; 
3. Generalized Watson's theorem:

$$
\begin{aligned}
& F_{a_{1}, \ldots, a_{j}, a_{j+1}, \ldots, a_{k}}^{\mathcal{O}}\left(\theta_{1}, \ldots, \theta_{j}, \theta_{j+1}, \ldots, \theta_{k}\right) \\
& \quad=S_{a_{j}, a_{j+1}}^{b_{j}, b_{j+1}}\left(\theta_{j}-\theta_{j+1}\right) F_{a_{1}, \ldots, b_{j+1}, b_{j}, \ldots, a_{k}}^{\mathcal{O}}\left(\theta_{1}, \ldots, \theta_{j+1}, \theta_{j}, \ldots, \theta_{k}\right)
\end{aligned}
$$

4. Locality:

$$
F_{a_{1}, \ldots, a_{k-1}, a_{k}}^{\mathcal{O}}\left(\theta_{1}, \ldots, \theta_{k-1}, \theta_{k}+2 \pi i\right)=(-1)^{f_{\mathcal{O}} f_{\Psi}} e^{2 \pi i \omega(\mathcal{O}, \Psi)} F_{a_{k}, a_{1}, \ldots, a_{k-1}}^{\mathcal{O}}\left(\theta_{k}, \theta_{1}, \ldots, \theta_{k-1}\right),
$$

where $f_{\mathcal{O}}$ is 1 if $\mathcal{O}$ is fermionic, 0 if it is bosonic, $\Psi$ is the fundamental field associated to the particle $a_{k}$, and $\omega(\mathcal{O}, \Psi)$ is the semi-locality index (or mutual locality index) of $\mathcal{O}$ with respect to $\Psi$ (it will be defined in Subsection 3.3);

5. Kinematic pole: as function of the variable $\theta_{n}$, there are poles at $\theta_{j}+i \pi$ for $j \in\{1, \ldots, k-1\}$, with residue

$$
\begin{aligned}
& i F_{a_{1}, \ldots, a_{k}}^{\mathcal{O}}\left(\theta_{1}, \ldots, \theta_{k}\right) \sim C_{a_{k}, b_{j}} \frac{F_{a_{1}, \ldots, \hat{a}_{j}, \ldots, a_{k-1}}\left(\theta_{1}, \ldots, \hat{\theta}_{j}, \ldots, \theta_{k-1}\right)}{\theta_{k}-\theta_{j}-i \pi} \\
& \quad \times\left(\delta_{a_{1}}^{b_{1}} \cdots \delta_{a_{j-1}}^{b_{j-1}} S_{a_{j+1}, a_{j}}^{b_{j+1}, c_{j}}\left(\theta_{j+1}-\theta_{j}\right) S_{a_{j+2}, c_{j}}^{b_{j+2}, c_{j+1}}\left(\theta_{j+2}-\theta_{j}\right) \cdots S_{a_{k-1}, c_{k-3}}^{b_{k-1}, b_{j}}\left(\theta_{k-1}-\theta_{j}\right)\right. \\
& \quad-(-1)^{f_{\mathcal{O}} f_{\Psi}} e^{2 \pi i \omega(\mathcal{O}, \Psi)} \delta_{a_{k-1}}^{b_{k-1}} \cdots \delta_{a_{j+1}}^{b_{j+1}} S_{a_{j}, a_{j-1}}^{c_{j}, b_{j-1}}\left(\theta_{j}-\theta_{j-1}\right) S_{c_{j}, a_{j-2}}^{c_{j-1}, b_{j-2}} \\
& \left.\quad \times\left(\theta_{j}-\theta_{j-2}\right) \cdots S_{c_{3}, a_{1}}^{b_{j}, b_{1}}\left(\theta_{j}-\theta_{1}\right)\right),
\end{aligned}
$$

where a hat means omission of the argument, and $C_{a_{k}, b_{j}}$ is the conjugation matrix.

6. Bound-state poles: there are additional poles in the strip $0<\operatorname{Im}\left(\theta_{i}-\theta_{j}\right)<\pi$ if bound states are present, and these are the only poles in that strip.

Form factors can in turn be used to obtain a large-distance expansion of two-point correlation functions of local fields:

$$
\begin{aligned}
\left\langle\operatorname{vac}\left|\mathcal{O}_{1}(x, t) \mathcal{O}_{2}(0,0)\right| \operatorname{vac}\right\rangle= & \sum_{k=0}^{\infty} \sum_{a_{1}, \ldots, a_{k}} \int \frac{d \theta_{1} \cdots d \theta_{k}}{k !} e^{-i t \sum_{j} E_{j}+i x \sum_{j} p_{j}} \\
& \times\left\langle\operatorname{vac}|\mathcal{O}(0,0)| \theta_{1}, \ldots, \theta_{k}\right\rangle_{a_{1}, \ldots, a_{k}} a_{1}, \ldots, a_{k}\left\langle\theta_{1}, \ldots, \theta_{k}|\mathcal{O}(0,0)| \operatorname{vac}\right\rangle .
\end{aligned}
$$

A large-distance expansion is effectively obtained by shifting all rapidity variables by $\pi / 2$ in the positive imaginary direction, and by using relativistic invariance. This gives a formula which looks as above, but with the replacement $e^{-i t \sum_{j} E_{j}+i x \sum_{j} p_{j}} \mapsto e^{-r \sum_{j} m_{a_{j}} \cosh \left(\theta_{j}\right)}$ where $r=\sqrt{x^{2}-t^{2}}$. It turns out that this is numerically extremely efficient in most integrable models that were studied.

\section{$3 \quad$ Finite temperature correlation functions}

\subsection{Traces}

Physical correlation functions at finite temperature are obtained by taking a statistical average of quantum averages, with Boltzmann weights $e^{-\beta E}$ where $E$ is the energy of the quantum state and $\beta$ is the inverse temperature. They are then represented by traces over the Hilbert space:

$$
\langle\langle\mathcal{O}(x, t) \cdots\rangle\rangle_{\beta}=\frac{\operatorname{Tr}\left[e^{-\beta H} \mathcal{O}(x, t) \cdots\right]}{\operatorname{Tr}\left[e^{-\beta H}\right]} .
$$


Since all matrix elements of local fields are known in many integrable models, it would seem appropriate to write the trace as an explicit sum over all states of the Hilbert space, and to introduce resolutions of the identity between operators inside the trace, in order to evaluate finite-temperature correlation functions. However, this method does not account correctly for the fact that at finite temperature, states that contribute to the trace are very far from the vacuum. Yet, it turned out to give good results in the case of correlation functions with only one operator $[33,34,32]$.

\subsection{Quantization on the circle}

On the other hand, traces as above can be represented by vacuum expectation values on the Hilbert space $\mathcal{H}_{\beta}$ of quantization on the circle of circumference $\beta$. Indeed, a consequence of the imaginary-time formalism [12] is the Kubo-Martin-Schwinger (KMS) identity [13, 14],

$$
\langle\langle\mathcal{O}(x, t) \cdots\rangle\rangle_{\beta}=(-1)^{f_{\mathcal{O}}}\langle\langle\mathcal{O}(x, t-i \beta) \cdots\rangle\rangle_{\beta},
$$

where $(-1)^{f_{\mathcal{O}}}$ is a sign accounting for the statistics of $\mathcal{O}$ (it is negative for fermionic operators and positive for bosinic operators), and where the dots $(\cdots)$ represent local fields (that are also local with respect to $\mathcal{O}$ ) at time $t$ and at positions different from $x$. Then, finite-temperature correlation functions can be written as

$$
\langle\langle\mathcal{O}(\tau, i \mathrm{x}) \cdots\rangle\rangle_{\beta}=\left(e^{i \pi s / 2} \cdots\right)_{\beta}\langle\operatorname{vac}|\hat{\mathcal{O}}(\mathrm{x}, \tau) \cdots| \operatorname{vac}\rangle_{\beta},
$$

where $s$ is the spin of $\mathcal{O}$, and there are factors $e^{-i \pi s / 2}$ for all operators in the correlation function. The operator $\hat{\mathcal{O}}(\mathrm{x}, \tau)$ is the corresponding operator acting on the Hilbert space $\mathcal{H}_{\beta}$ of quantization on the circle, with space variable $\mathrm{x}$ (parameterizing the circle of circumference $\beta$ ) and Euclidean time variable $\tau$ (on the line). The vector $|v a c\rangle_{\beta}$ is the vacuum in this Hilbert space. Below, we will mostly be interested in fermionic models, that is, models with a "fundamental" fermion field (which creates from the vacuum single-particle states). For such models, one can think of at least two sectors in the quantization on the circle: Neveu-Schwartz (NS) and Ramond (R), where the fundamental fermion fields are anti-periodic and periodic, respectively, around the circle. The trace (3.1) with insertion of operators that are local with respect to the fermion fields naturally corresponds to the NS sector due to the KMS identity. This is the sector with the lowest vacuum energy.

The representation (3.3) immediately leads to a large-distance expansion of finite-temperature correlation functions, through insertion of the resolution of the identity on the Hilbert space $\mathcal{H}_{\beta}$ :

$$
\begin{aligned}
{ }_{\beta}\left\langle\operatorname{vac}_{1}|\hat{\mathcal{O}}(\mathrm{x}, \tau) \hat{\mathcal{O}}(0,0)| \operatorname{vac}_{2}\right\rangle_{\beta}= & \sum_{k=0}^{\infty} \sum_{n_{1}, \ldots, n_{k}} \frac{e^{\sum_{j} n_{j} \frac{2 \pi i \mathrm{x}}{\beta}+\left(\Delta \mathcal{E}-E_{n_{1}, \ldots, n_{k}}\right) \tau}}{k !} \\
& \times{ }_{\beta}\left\langle\operatorname{vac}_{1}|\hat{\mathcal{O}}(0,0)| n_{1}, \ldots, n_{k}\right\rangle_{\beta}{ }_{\beta}\left\langle n_{1}, \ldots, n_{k}|\hat{\mathcal{O}}(0,0)| \operatorname{vac}_{2}\right\rangle_{\beta},
\end{aligned}
$$

where the eigenstates of the momentum operator and of the Hamiltonian on the circle are parametrized by discrete variables $n_{j}$ 's. The vacua $\left|\operatorname{vac}_{1}\right\rangle_{\beta}$ and $\left|\operatorname{vac}_{2}\right\rangle_{\beta}$ may be in different sectors, and these sectors may be different than the sector where the excited states $\left|n_{1}, \ldots, n_{k}\right\rangle_{\beta}$ lie (this situation occurs when considering semi-local operators as is recalled in the Subsection 3.3 below). The quantity $\Delta \mathcal{E}$ is the difference between the vacuum energies of the vacuum state $\left|\operatorname{vac}_{1}\right\rangle_{\beta}$ and of the vacuum above which the states $\left|n_{1}, \ldots, n_{k}\right\rangle_{\beta}$ are constructed. The states $\left|n_{1}, \ldots, n_{k}\right\rangle_{\beta}$ and the excitation energies $E_{n_{1}, \ldots, n_{k}}$ may also depend on additional discrete parameters (quantum numbers, particle types), on which one has to sum as well.

This form is valid for any integrable model on the circle. However, this Hilbert space has a very complicated structure, even in integrable quantum field theory; for instance the energy 
levels $E_{n_{1}, \ldots, n_{k}}$ are not known in closed form. Also, there is yet no known procedure in general integrable quantum field theory for evaluating form factors on this Hilbert space. Moreover, this representation does not provide large-time (real time) expansions, since it inherently gives finite-temperature correlation functions in Euclidean time.

\subsection{Semi-locality: $U(1)$ twist fields}

If the model we are considering has internal global symmetries, then there are local twist fields associated to them. Twist fields are of interest, because they usually correspond to some order parameter. We will clarify the correspondence between order/disorder parameters in the quantum Ising chain and twist fields in the Section 7.6. The first appearances of certain twist fields in the context of the Ising statistical model can be found in [40, 41], but we are going to describe twist fields here in more general terms (see, for instance, the lecture notes [42]).

Twist fields are not local with respect to the fundamental fields associated to a given particle (but are with respect to the energy density). If the symmetry to which they are associated is $U(1)$ or a subgroup of it (and if the fundamental field transform by multiplication by a phase), then the twist fields are said to be semi-local with respect to the fundamental field. In the quantization scheme on the line, a twist field, which we will generically denote by $\sigma$, gives rise to a pair of operators, which we will denote by $\sigma_{+}(x, t)$ and $\sigma_{-}(x, t)$, having a cut towards the right (positive $x$ direction) and towards the left (negative $x$ direction), respectively. These operators lead to the same correlation functions at zero temperature.

When considering correlation functions at finite temperature, things are more subtle. The exact shape of the cuts are unimportant, but it is important if the cut is towards the right or towards the left. This is because the insertion of an operator $\sigma_{ \pm}(x, t)$ that is semi-local with respect to the fundamental field $\Psi(x, t)$ may affect the vacuum sector in the correspondence to expectation values in the quantization on the circle. Semi-locality can be expressed through the exchange relations

$$
\Psi(x, t) \sigma_{+}\left(x^{\prime}, t\right)=(-1)^{f_{\Psi} f_{\sigma}} e^{-2 \pi i \omega \Theta\left(x-x^{\prime}\right)} \sigma_{+}\left(x^{\prime}, t\right) \Psi(x, t) \quad\left(x \neq x^{\prime}\right)
$$

and

$$
\Psi(x, t) \sigma_{-}\left(x^{\prime}, t\right)=(-1)^{f_{\Psi} f_{\sigma}} e^{2 \pi i \omega \Theta\left(x^{\prime}-x\right)} \sigma_{-}\left(x^{\prime}, t\right) \Psi(x, t) \quad\left(x \neq x^{\prime}\right),
$$

where $\Theta(x)$ is Heaviside's step function and $\omega$ is the semi-locality index associated to the pair $(\Psi, \sigma)$. Taking here the fundamental field to be fermionic (because this is what will be of interest in the following - the case of bosonic fundamental fields is straightforward to work out), it is a simple matter to generalise the KMS identity to (using $f_{\Psi}=1$ )

$$
\begin{aligned}
& \left\langle\left\langle\Psi(x, t) \sigma_{+}\left(x^{\prime}, t\right) \cdots\right\rangle\right\rangle_{\beta}= \begin{cases}-e^{-2 \pi i \omega}\left\langle\left\langle\Psi(x, t-i \beta) \sigma_{+}\left(x^{\prime}, t\right) \cdots\right\rangle\right\rangle_{\beta} & (x \rightarrow \infty), \\
-\left\langle\left\langle\Psi(x, t-i \beta) \sigma_{+}\left(x^{\prime}, t\right) \cdots\right\rangle\right\rangle_{\beta} & (x \rightarrow-\infty),\end{cases} \\
& \left\langle\left\langle\Psi(x, t) \sigma_{-}\left(x^{\prime}, t\right) \cdots\right\rangle\right\rangle_{\beta}= \begin{cases}-\left\langle\left\langle\Psi(x, t-i \beta) \sigma_{-}\left(x^{\prime}, t\right) \cdots\right\rangle\right\rangle_{\beta} & (x \rightarrow \infty), \\
-e^{2 \pi i \omega}\left\langle\left\langle\Psi(x, t-i \beta) \sigma_{-}\left(x^{\prime}, t\right) \cdots\right\rangle\right\rangle_{\beta} & (x \rightarrow-\infty),\end{cases}
\end{aligned}
$$

where the dots $(\cdots)$ represent fields that are local with respect to the fermion field $\Psi$, at time $t$ and at positions different from $x$. Then, in the correspondence of the trace with a vacuum expectation value in the quantization on the circle, one of the vacua will be in a different sector, in accordance with these quasi-periodicity relations. Denoting by $\left|\operatorname{vac}_{\nu}\right\rangle_{\beta}$ the vacuum in the quantization on the circle with quasi-periodicity condition $\Psi \mapsto e^{-2 \pi i \nu} \Psi$ around the circle in the positive space (x) direction, we have

$$
\left\langle\left\langle\sigma_{+}(\tau, i \mathrm{x}) \cdots\right\rangle\right\rangle_{\beta}=\left(e^{i \pi s / 2} \cdots\right)_{\beta}\left\langle\operatorname{vac}_{\frac{1}{2}+\omega}|\hat{\sigma}(\mathrm{x}, \tau) \cdots| \operatorname{vac}_{\mathrm{NS}}\right\rangle_{\beta}
$$


and

$$
\left\langle\left\langle\sigma_{-}(\tau, i \mathrm{x}) \cdots\right\rangle\right\rangle_{\beta}=\left(e^{i \pi s / 2} \cdots\right)_{\beta}\left\langle\operatorname{vac}_{\mathrm{NS}}|\hat{\sigma}(\mathrm{x}, \tau) \cdots| \operatorname{vac}_{\frac{1}{2}-\omega}\right\rangle_{\beta},
$$

where $\left|\operatorname{vac}_{\mathrm{NS}}\right\rangle_{\beta}=\left|\operatorname{vac}_{\frac{1}{2}}\right\rangle_{\beta}$ is the NS vacuum, and where the dots $(\cdots)$ represent operators that are local with respect to the fundamental fermion fields.

With many insertions of semi-local operators, similar phenomena arise. This change of the vacuum sector has an important effect: under translation in the $x$ direction, the insertion of an operator $\sigma_{ \pm}(x, t)$ inside a trace produces an additional real exponential factor, due to the difference between the vacuum energies of the different sectors; that is, the trace is not translation invariant. It is convenient to represent this lack of translation invariance of traces, in the case where many semi-local operators are multiplied, by considering "modified" transformation properties of this product of semi-local operators. Consider the product $\sigma_{\eta_{1}}^{\omega_{1}}\left(x_{1}, t_{1}\right) \cdots \sigma_{\eta_{k}}^{\omega_{k}}\left(x_{k}, t_{k}\right)$ where $\eta_{i}= \pm$ and we have indicated explicitly the semi-locality indices $\omega_{i}$. Then, inside traces at temperature $\beta$ with insertion of operators that are local with respect to the fundamental fermion field, we have

$$
\begin{aligned}
& e^{-i P \delta} \sigma_{\eta_{1}}^{\omega_{1}}\left(x_{1}, t_{1}\right) \cdots \sigma_{\eta_{k}}^{\omega_{k}}\left(x_{k}, t_{k}\right) e^{i P \delta}=e^{-\Delta \mathcal{E} \delta} \sigma_{\eta_{1}}^{\omega_{1}}\left(x_{1}+\delta, t_{1}\right) \cdots \sigma_{\eta_{k}}^{\omega_{k}}\left(x_{k}+\delta, t_{k}\right), \\
& \Delta \mathcal{E}=\mathcal{E}\left[\frac{1}{2}+\sum_{\substack{i=1 \\
\eta_{i}=+}}^{k} \omega_{i}\right]-\mathcal{E}\left[\frac{1}{2}-\sum_{\substack{i=1 \\
\eta_{i}=-}}^{k} \omega_{i}\right]
\end{aligned}
$$

where $\mathcal{E}[\nu]$ is the energy of the vacuum $\left|\operatorname{vac}_{\nu}\right\rangle_{\beta}$.

\section{A space of "finite-temperature states" in integrable quantum field theory}

In [2], it was suggested that the difficulties in obtaining large-distance or large-time expansions of finite-temperature correlation functions can be overcome by constructing a finite-temperature Hilbert space in terms of objects with nice analytic structure, in analogy with the zero-temperature case. The program was carried out explicitly in the free massive Majorana theory (considering, in particular, "interacting" twist fields). As we said in the introduction, the idea of a finite-temperature Hilbert space is far from new, but it is in [2] that it was first developed in the context of an integrable quantum field theory.

\subsection{General idea}

The idea of the construction is simply to consider the space $\mathcal{L}$ of endomorphisms of $\mathcal{H}$ as a Hilbert space with inner product structure

$$
(A, B)=\frac{\operatorname{Tr}\left(e^{-\beta H} A^{\dagger} B\right)}{\operatorname{Tr}\left(e^{-\beta H}\right)} .
$$

This Hilbert space is known as the Liouville space [43]. Note that

$$
(A, B)^{*}=(B, A) .
$$

There is then a (generically) one-to-two mapping from $\operatorname{End}(\mathcal{H})$ to $\operatorname{End}(\mathcal{L})$ : to each operator $C$ acting on $\mathcal{H}$, there are two operators, $\phi_{L}(C)$ and $\phi_{R}(C)$, acting on $\mathcal{L}$, defined respectively by left action and by right action of $C$ as follows:

$$
\left(A, \phi_{L}(C) B\right)=\frac{\operatorname{Tr}\left(e^{-\beta H} A^{\dagger} C B\right)}{\operatorname{Tr}\left(e^{-\beta H}\right)}, \quad\left(A, \phi_{R}(C) B\right)=\frac{\operatorname{Tr}\left(e^{-\beta H} A^{\dagger} B C\right)}{\operatorname{Tr}\left(e^{-\beta H}\right)} .
$$


In particular, if $Q$ is a generator of a symmetry transformation on $\mathcal{H}$, then $\phi_{L}(Q)-\phi_{R}(Q)$ is the generator on $\mathcal{L}$. The set of all operators on $\mathcal{L}$ that are in the image of at least one of $\phi_{L}$ or $\phi_{R}$ will be denoted $\operatorname{End}_{L R}(\mathcal{L})$.

The main power of this construction, from our viewpoint, is the possibility to obtain largedistance or large-time expansions at finite temperature, in analogy with the zero-temperature case, using a resolution of the identity on the space $\mathcal{L}$. Indeed, suppose we have a complete set of orthonormal operators $D\left(\theta_{1}, \ldots, \theta_{k}\right), \theta_{1}>\cdots>\theta_{k} \in \mathbb{R}, k \in \mathbb{N}$ :

$$
\left(D\left(\theta_{1}, \ldots, \theta_{k}\right), D\left(\theta_{1}^{\prime}, \ldots, \theta_{l}^{\prime}\right)\right)=\delta_{k, l} \delta\left(\theta_{1}-\theta_{1}^{\prime}\right) \cdots \delta\left(\theta_{k}-\theta_{k}^{\prime}\right) .
$$

Then, we can decompose any inner product as a sum of products of inner products:

$$
(A, B)=\sum_{k=0}^{\infty} \int_{\theta_{1}>\cdots>\theta_{k}} d \theta_{1} \cdots d \theta_{k}\left(A, D\left(\theta_{1}, \ldots, \theta_{k}\right)\right)\left(D\left(\theta_{1}, \ldots, \theta_{k}\right), B\right) .
$$

This is a non-trivial relation equating a trace on the left-hand side to a sum of products of traces on the right-hand side.

\subsection{A natural basis}

A natural complete set of operators in integrable quantum field theory can be obtained as follows (more precisely, one should consider an appropriate completion of the set below). First, define a larger set of particle types $E_{\mathcal{L}}=E \oplus E$, the elements being the couples $\alpha=(a, \epsilon)$ for $a \in E$ and $\epsilon= \pm$. For notational convenience, define $\bar{Z}_{\alpha}=\bar{Z}_{a}$ if $\epsilon=+$ and $\bar{Z}_{\alpha}=Z^{a}$ if $\epsilon=-$. Then, we have a complete set with

$$
D_{\alpha_{1}, \ldots, \alpha_{k}}\left(\theta_{1}, \ldots, \theta_{k}\right)=\bar{Z}_{\alpha_{1}}\left(\theta_{1}\right) \cdots \bar{Z}_{\alpha_{k}}\left(\theta_{k}\right)
$$

for

$$
\alpha_{i} \in E_{\mathcal{L}}, \quad \theta_{1}>\cdots>\theta_{k} \in \mathbb{R}, \quad k \in \mathbb{N} .
$$

In fact, it will be convenient to define the operators $D_{\alpha_{1}, \ldots, \alpha_{k}}\left(\theta_{1}, \ldots, \theta_{k}\right)$ for any ordering of the rapidities, and to define them as being exactly zero when two rapidities collide (in order to avoid overlap with operators with smaller $k$ ):

$$
D_{\alpha_{1}, \ldots, \alpha_{k}}\left(\theta_{1}, \ldots, \theta_{k}\right)=\left\{\begin{array}{lll}
\bar{Z}_{\alpha_{1}}\left(\theta_{1}\right) \cdots \bar{Z}_{\alpha_{k}}\left(\theta_{k}\right), & \theta_{i} \neq \theta_{j} & \forall i \neq j, \\
0, & \theta_{i}=\theta_{j} & \text { for some } i \neq j .
\end{array}\right.
$$

These operators will form a very useful set if the matrix elements (with $\mathcal{O}(x, t)$ a local operator and $\mathcal{O}^{\dagger}(x, t)$ its Hermitian conjugate on $\left.\mathcal{H}\right)$

$$
\left(\mathcal{O}^{\dagger}(x, t), D_{\alpha_{1}, \ldots, \alpha_{k}}\left(\theta_{1}, \ldots, \theta_{k}\right)\right)
$$

have simple analytical properties; for instance, if the value of this function for a certain ordering of the rapidities is the one obtained by analytical continuation from that for a different ordering. Then, it may be possible to write down equations similar to the zero-temperature form factor equations (what we will call the "finite-temperature form factor equations") and to solve them. Two clues suggest that this may be so, at least for models with diagonal scattering (see below): first, these objects specialise to the zero-temperature form factors when the temperature is sent to zero and all signs $\epsilon_{i}$ 's are set to + , and second, in [2] the finite-temperature form factor equations were indeed written and solved in the free massive Majorana theory (this will be recalled in Section 7). 
Although the operators (4.2) form a complete set, they are not orthonormal. In the case of diagonal scattering (and this is the only case we will consider from now on)

$$
S_{a_{1}, a_{2}}^{b_{1}, b_{2}}(\theta)=\delta_{a_{1}}^{b_{1}} \delta_{a_{2}}^{b_{2}} S_{a_{1}, a_{2}}(\theta)
$$

(without summation over repeated indices), it is possible to write down all inner products in a simple way, using the Zamolodchikov-Faddeev algebra (2.1) and the cyclic property of the trace:

$$
\left(D_{\alpha_{1}, \ldots, \alpha_{k}}\left(\theta_{1}, \ldots, \theta_{k}\right), D_{\alpha_{1}^{\prime}, \ldots, \alpha_{l}^{\prime}}\left(\theta_{1}^{\prime}, \ldots, \theta_{l}^{\prime}\right)\right)=\delta_{k, l} \prod_{i=1}^{k} \frac{\epsilon_{i}^{1-f_{a_{i}}} \delta_{a_{i}, a_{i}^{\prime}} \delta\left(\theta_{i}-\theta_{i}^{\prime}\right)}{1-(-1)^{f a_{i}} e^{-\epsilon_{i} \beta m_{a_{i}} \cosh \theta_{i}}}
$$

where from unitarity, $(-1)^{f_{a}} \equiv S_{a, a}(0)= \pm 1$ (this corresponds to the statistics of the particle of type $a$, as an asymptotically free particle). Here, we have assumed the ordering (4.1) for both members of the inner product.

Note that simple "crossing" relations hold for operators in $\operatorname{End}_{R L}(\mathcal{L})$ :

$$
\begin{aligned}
& \left(D_{\alpha_{1}, \ldots, \alpha_{k}}\left(\theta_{1}, \ldots, \theta_{k}\right), \phi_{L}(A) D_{\alpha_{1}^{\prime}, \ldots, \alpha_{l}^{\prime}}\left(\theta_{1}^{\prime}, \ldots, \theta_{l}^{\prime}\right)\right) \\
& =e^{\epsilon_{l}^{\prime} \beta m_{a_{l}^{\prime}} \cosh \theta_{l}^{\prime}}\left(D_{\alpha_{1}, \ldots, \alpha_{k}, \bar{\alpha}_{l}^{\prime}}\left(\theta_{1}, \ldots, \theta_{k}, \theta_{l}^{\prime}\right), \phi_{L}(A) D_{\alpha_{1}^{\prime}, \ldots, \alpha_{l-1}^{\prime}}\left(\theta_{1}^{\prime}, \ldots, \theta_{l-1}^{\prime}\right)\right) \quad\left(\theta_{l}^{\prime} \neq \theta_{i} \forall i\right)
\end{aligned}
$$

and

$$
\begin{aligned}
& \left(D_{\alpha_{1}, \ldots, \alpha_{k}}\left(\theta_{1}, \ldots, \theta_{k}\right), \phi_{R}(A) D_{\alpha_{1}^{\prime}, \ldots, \alpha_{l}^{\prime}}\left(\theta_{1}^{\prime}, \ldots, \theta_{l}^{\prime}\right)\right) \\
& \quad=\left(D_{\bar{\alpha}_{1}^{\prime}, \alpha_{1}, \ldots, \alpha_{k}}\left(\theta_{1}^{\prime}, \theta_{1}, \ldots, \theta_{k}\right), \phi_{R}(A) D_{\alpha_{2}^{\prime}, \ldots, \alpha_{l}^{\prime}}\left(\theta_{2}^{\prime}, \ldots, \theta_{l}^{\prime}\right)\right) \quad\left(\theta_{1}^{\prime} \neq \theta_{i} \forall i\right),
\end{aligned}
$$

where $\overline{(a, \epsilon)}=(a,-\epsilon)$.

\subsection{Finite-temperature form factor expansion}

Inverting (4.3), and using the fact that the operators (4.2) are eigenoperators of both the Hamiltonian and the momentum operator, we get a spectral decomposition for two-point functions $\left\langle\left\langle\mathcal{O}_{1}(x, t) \mathcal{O}_{2}(0,0)\right\rangle\right\rangle_{\beta}$ (finite-temperature form factor expansion). In order to simplify the discussion, we assume that $x>0$. This can always be achieved by taking complex conjugation if necessary: $\left\langle\left\langle\mathcal{O}_{1}(x, t) \mathcal{O}_{2}(0,0)\right\rangle\right\rangle_{\beta}^{*}=\left\langle\left\langle\mathcal{O}_{2}^{\dagger}(0,0) \mathcal{O}_{1}^{\dagger}(x, t)\right\rangle\right\rangle_{\beta}$ (a slightly different formulation holds with $x<0)$. We have

$$
\begin{aligned}
& \left\langle\left\langle\mathcal{O}_{1}(x, t) \mathcal{O}_{2}(0,0)\right\rangle\right\rangle_{\beta} \\
& =e^{\Delta \mathcal{E} x} \sum_{k=0}^{\infty} \sum_{\alpha_{1}, \ldots, \alpha_{k}} \int_{\left\{\operatorname{Im}\left(\theta_{j}\right)=\epsilon_{j} 0^{+}\right\}} \frac{d \theta_{1} \cdots d \theta_{k} \prod_{j=1}^{k} \epsilon_{j}^{1-f_{a_{j}}} e^{\sum_{j=1}^{k} \epsilon_{j}\left(i m_{a_{j}} x \sinh \theta_{j}-i m_{a_{j}} t \cosh \theta_{j}\right)}}{k ! \prod_{j=1}^{k}\left(1-(-1)^{f_{a_{j}}} e^{-\epsilon_{j} \beta m_{a_{j}} \cosh \theta_{j}}\right)} \\
& \quad \times F_{\alpha_{1}, \ldots, \alpha_{k}}^{\mathcal{O}_{1}}\left(\theta_{1}, \ldots, \theta_{k} ; \beta\right) F_{-\alpha_{k}, \ldots,-\alpha_{1}}^{\mathcal{O}_{2}}\left(\theta_{k}, \ldots, \theta_{1} ; \beta\right),
\end{aligned}
$$

where we have defined finite-temperature form factors as the normalised matrix elements ${ }^{1}$ :

$$
\begin{aligned}
& F_{\alpha_{1}, \ldots, \alpha_{k}}^{\mathcal{O}}(\left.\theta_{1}, \ldots, \theta_{k} ; \beta\right) \\
& \quad=\prod_{i=1}^{n}\left[\epsilon_{i}^{1-f_{a_{i}}}\left(1-(-1)^{f_{a_{i}}} e^{-\epsilon_{i} \beta m_{a_{i}} \cosh \theta_{i}}\right)\right]\left(\mathcal{O}^{\dagger}(0,0), D_{\alpha_{1}, \ldots, \alpha_{k}}\left(\theta_{1}, \ldots, \theta_{k}\right)\right) .
\end{aligned}
$$

\footnotetext{
${ }^{1}$ Note that by definition of the basis of states in $\mathcal{L}$, the function $F_{\alpha_{1}, \ldots, \alpha_{n}}^{\mathcal{O}}\left(\theta_{1}, \ldots, \theta_{n} ; \beta\right)$ has no delta-function contributions at colliding rapidities.
} 
This normalisation is for later convenience (one may call it the "free field" normalisation). It leads in particular to the following identity, which we have used to write the expansion:

$$
\left(F_{\alpha_{1}, \ldots, \alpha_{k}}^{\mathcal{O}_{2}^{\dagger}}\left(\theta_{1}^{*}, \ldots, \theta_{k}^{*} ; \beta\right)\right)^{*}=F_{-\alpha_{k}, \ldots,-\alpha_{1}}^{\mathcal{O}_{2}}\left(\theta_{k}, \ldots, \theta_{1} ; \beta\right)
$$

which essentially follows from (6.2) below. In the expansion (4.6), we have symmetrised over the orderings of rapidities. The quantity $\Delta \mathcal{E}$ is non-zero whenever $\mathcal{O}_{1}$ is a twist field $\sigma_{\eta_{1}}^{\omega_{1}}$, and is given by

$$
\Delta \mathcal{E}= \begin{cases}\mathcal{E}\left[\frac{1}{2}+\omega_{1}\right]-\mathcal{E}\left[\frac{1}{2}\right] & \left(\eta_{1}=+\right), \\ \mathcal{E}\left[\frac{1}{2}\right]-\mathcal{E}\left[\frac{1}{2}-\omega_{1}\right] & \left(\eta_{1}=-\right),\end{cases}
$$

where $\mathcal{E}[\nu]$ is the energy of the vacuum $\left|\operatorname{vac}_{\nu}\right\rangle_{\beta}$ (see the discussion around (3.9)).

Some comments are due:

- As we said, the factor $e^{\Delta \mathcal{E} x}$ is present whenever the operator $\mathcal{O}_{1}$ is semi-local, $\mathcal{O}_{1}=\sigma_{\eta}^{\omega}$, with respect to the fundamental fermion field. It is as in (3.9) with $k=1$, and with $\omega_{1}=\omega$ and $\eta_{1}=\eta$ the semi-locality index and cut direction, respectively, of $\mathcal{O}_{1}$. It occurs because the operators $\bar{Z}_{\alpha}(\theta)$ can be expressed through integrals of the fundamental fermion field. To be more precise, in order to deduce it from the discussion around (3.9), one has to assume that although these integrals extend to $\pm \infty$ in the $x$ direction, they only produce excited states, without changing the sector. The presence of this exponential factor can in fact be shown for the finite-temperature form factors of the order field $\sigma_{ \pm}^{\frac{1}{2}}$ in the Majorana theory. Indeed, as we said, in the Majorana theory the traces $F_{\alpha_{1}, \ldots, \alpha_{n}}^{\sigma^{\frac{1}{2}}}\left(\theta_{1}, \ldots, \theta_{n} ; \beta\right)$ were shown in [2] to satisfy a set of recursive relations which ultimately relate them to the one-point function (in the case of the order field). Slightly generalising the derivation to include an $x$ and $t$ dependence, this accounts for the phase factors above. On the other hand, the one-point function of a twist field is not translation invariant, as is clear from (3.7) and (3.8), the transformation property being as in (3.9). This is what accounts for the real exponential factor (this factor was missing in [2], because the one-point function was considered translation invariant).

- When both $\mathcal{O}_{1}$ and $\mathcal{O}_{2}$ are semi-local with respect to the fundamental fermion fields, the finite-temperature form factor expansion (4.6) is valid only when the cut of $\mathcal{O}_{1}$ extends towards the right (positive $x$ direction) and that of $\mathcal{O}_{2}$ extends towards the left (negative $x$ direction). This will be justified in the Subsection 4.4. Note that with this prescription on the directions of the cuts, one produces the correlation functions

$$
{ }_{\beta}\left\langle\operatorname{vac}_{\frac{1}{2}+\omega_{1}}\left|\hat{\mathcal{O}}_{1}(\mathrm{x}, \tau) \hat{\mathcal{O}}_{2}(0,0)\right| \operatorname{vac}_{\frac{1}{2}-\omega_{2}}\right\rangle_{\beta}
$$

when written in the quantization on the circle, with $x=\tau$ and $t=i \mathrm{x}$. This is a restriction, as not all vacua on $\mathcal{H}_{\beta}$ can be obtained. For instance, one would like to evaluate correlation functions of twist fields with the NS vacuum. This restriction will be lifted in Section 5 .

- Had we not put a small imaginary part to the rapidities in the integrals, the expansion (4.6) would have been plagued by singularities: as the rapidity associated to a particle of type $(a, \epsilon)$ becomes equal to that associated to a particle of type $(a,-\epsilon)$, poles are expected to appear in the finite-temperature form factors (kinematic poles). This expectation comes from the intuition from zero-temperature form factors, and from the fact that these singularities indeed occur in the finite-temperature form factors of twist fields in the Majorana 
theory, as was calculated in [2]. There it was shown that a proper solution is obtained by slightly deforming the integration contours as indicated above:

$$
\operatorname{Im}\left(\theta_{j}\right)=\epsilon_{j} 0^{+} .
$$

That this is the right prescription still in the interacting case will be argued in Subsection 4.4 .

- It is important to realise that the expansion (4.6) is not directly a large-distance or a largetime expansion. But it can be made so as follows (for large-time expansions, this requires some work). First, with further displacement of the integration contours in the directions of (4.8), more precisely with $\operatorname{Im}\left(\theta_{j}\right)=\epsilon_{j} \pi / 2$, the expansion (4.6) becomes an expansion at large $x^{2}-t^{2}$ (recall that we consider $x>0$ for simplicity). In order to perform this contour displacement, one needs to know about the analytical structure of the integrands; this will be briefly discussed in Subsection 4.4. Second, the integrals involved in (4.6) can be made convergent in time-like regions $t^{2}-x^{2}>0$ by deforming the contours in the following way:

$$
\begin{array}{ll}
|t|>|x|, t>0: & \operatorname{Im}\left(\theta_{j}\right)=-\operatorname{sgn}\left(\operatorname{Re}\left(\theta_{j}\right)\right) \epsilon_{j} 0^{+}, \\
|t|>|x|, t<0: & \operatorname{Im}\left(\theta_{j}\right)=\operatorname{sgn}\left(\operatorname{Re}\left(\theta_{j}\right)\right) \epsilon_{j} 0^{+} .
\end{array}
$$

These deformations necessitate the addition of residues coming from the kinematic poles. These residues will lead to powers of the time variable, which will need to be re-summed. Note that it was assumed in [27] that considering the contributions near the singularities at colliding rapidities, the expansion gives the leading in some semi-classical region, which should include a large-time limit $t^{2}-x^{2} \rightarrow \infty$. The full contour deformation should give a definite answer as to the large-time dynamics (work is in progress [31]).

- When calculating the spectral density (from the Fourier transform of the two-point function), the expansion (4.6) does produce an expansion with terms of lesser and lesser importance as the particle number is increased, at least for large enough energies. However, one does not have the situation where the spectral density is known exactly up to a certain energy depending on the number of particles considered, as happens at zero temperature. It would be very interesting to have a full analysis of the spectral density at finite temperature.

\subsection{From finite-temperature states to the quantization on the circle}

A great part of the structure of the finite-temperature form factor expansion can be understood according to the following idea.

Suppose that we have a model of quantum field theory; more precisely, let us consider a statistical field theory, on a space with Euclidean signature. Let us quantize it with a certain choice of space $x$ of infinite extent, and Euclidean time $t^{E}$. If we were starting from a Lorentzian quantum field theory, with real time $t$, we would just be considering the Wick rotated variable $t=-i t^{E}$. Then, the Hilbert space is the space of field configurations on $x$, with appropriate asymptotic conditions. On this space, we choose a vacuum $|v a c\rangle$ such that correlation functions are vacuum expectation values. Now suppose that a basis of states is chosen such that the generator of $x$ translations is diagonalised. The operator producing $x$ translations is unitary, of the form $e^{-i P x}$, where $P$ is the Hermitian generator. Suppose that the states are parametrised by the real eigenvalues $p$ of the operator $P$. Since space is of infinite extent, then $p$ takes all real values.

Then, formally, if we were to "analytically continue the theory" towards positive imaginary eigenvalues $p=i E$, the operator producing $x$ translations would have the form $e^{H x}$ for some Hermitian $H$ with eigenvalues $E$. The claim is that the operator $H$ is still the generator of $x$ 
translation, but now in a different quantization scheme (that is, on a different Hilbert space), where $x=\tau$ is the Euclidean time and $t^{E}=-\mathrm{x}$ is the space variable. Indeed, in that quantization scheme, the operator producing Euclidean time translations is $e^{H \tau}$ with $H$ the Hamiltonian (generator of time translations).

This formal analytical continuation has to be made more precise. Consider matrix elements of local operators $\left\langle\operatorname{vac}\left|\mathcal{O}\left(x, t^{E}\right)\right| p\right\rangle$ with states $|p\rangle$ of $P$-eigenvalue $p$. Then, this matrix element has singularities as function of $p$ on the positive imaginary axis, and the positions of these singularities exactly coincide with the eigenvalues of the Hamiltonian $H$ in the quantization scheme where $x$ is Euclidean time. Moreover, the analytical continuation of the matrix element towards these singularities gives the matrix element of the same operator in the quantization scheme where $x$ is Euclidean time. In relativistic quantum field theory, the singularities are branch cuts coming from the measure $\prod_{i} \sqrt{p_{i}^{2}+m_{i}^{2}}$ (with $p=\sum_{i} p_{i}$ ), and the statement about analytical continuation is just crossing symmetry. This claim was also verified to be true in the free Dirac theory on the Poincaré disk [44], where the singularities are poles and the residues must be taken.

In the case of present interest, our claim is that the "analytical continuation" of the Hilbert space $\mathcal{L}$ is nothing else than the Hilbert space $\mathcal{H}_{\beta}$ of quantization on the circle. This was verified explicitly in [2] in the free Majorana theory.

This claim is made relatively clear by comparing the finite-temperature form factor expansion (4.6) and the expansion in the quantization scheme on the circle (3.4), which must agree. The analytical continuation we talked about is obtained by shifting the contours of the rapidities $\theta_{j}$ by the amount $\epsilon_{j} i \pi / 2$ : then the exponential factors of (4.6) and of (3.4) indeed agree, under the identification $x=\tau, t=i \mathrm{x}$. This displacement of the contours can be performed while keeping all integrals convergent: we impose $x>0,|x|>|t|$ (space-like region) keeping $x$ and $t$ fixed, and make only at the end the analytical continuation $t=i \mathrm{x}$. Hence, we see that keeping the integrals convergent leads to keeping the operators time-ordered in the quantization on the circle. Note that it is here that the condition $x>0$ becomes important: the analytical conditions that define the finite-temperature form factors will be seen below as consequence of this analytical continuation, and depend, at least for twist fields, on our choice of sign of $x$. A different choice of sign would have required a shift in a different direction, and would have imposed different conditions on the finite-temperature form factors.

Of course, the series themselves must agree, but it is natural to assume that they agree individually for each term with a fixed number of excitations (at least this can be expected for integrable models, where there is no particle production - see section 6 for a discussion). Then, these terms will agree for all local fields if the following conditions are satisfied:

- The factor

$$
\frac{F_{\alpha_{1}, \ldots, \alpha_{k}}^{\mathcal{O}_{1}}\left(\theta_{1}, \ldots, \theta_{k} ; \beta\right) F_{-\alpha_{k}, \ldots,-\alpha_{1}}^{\mathcal{O}_{2}}\left(\theta_{k}, \ldots, \theta_{1} ; \beta\right)}{\prod_{j=1}^{k}\left(1-(-1)^{f_{a_{j}}} e^{-\epsilon_{j} \beta m_{a_{j}} \cosh \theta_{j}}\right)}
$$

can be written as

$$
\rho_{\alpha_{1}, \ldots, \alpha_{k}}\left(\theta_{1}, \ldots, \theta_{k}\right) \tilde{F}_{\alpha_{1}, \ldots, \alpha_{k}}^{\mathcal{O}_{1}}\left(\theta_{1}, \ldots, \theta_{k} ; \beta\right) \tilde{F}_{-\alpha_{k}, \ldots,-\alpha_{1}}^{\mathcal{O}_{2}}\left(\theta_{k}, \ldots, \theta_{1} ; \beta\right),
$$

where $\tilde{F}_{\alpha_{1}, \ldots, \alpha_{k}}^{\mathcal{O}}\left(\theta_{1}, \ldots, \theta_{k} ; \beta\right)$ does not have a pole in the strip $\operatorname{Im}\left(\theta_{j}\right) \in\left[0, \epsilon_{j} \pi / 2\right]$ for all local fields $\mathcal{O}$, and the measure $\rho_{\alpha_{1}, \ldots, \alpha_{k}}\left(\theta_{1}, \ldots, \theta_{k}\right)$ has poles at $\theta_{j}=q_{j}+\epsilon_{j} i \pi / 2$ for various real $q_{j}$ (more precisely, there are sets $s_{l}^{(k)}=\left\{q_{1}, \ldots, q_{k}\right\}$ for which the measure $\rho$ has poles at such positions, choosing an order: for instance, the pole as function of $\theta_{1}$, whose residue has a pole as function of $\theta_{2}$, etc.). 
- We have

$$
\sum_{j=1}^{k} m_{a_{j}} \cosh q_{j}=E_{n_{1}, \ldots, n_{k}}, \quad \sum_{j=1}^{k} m_{a_{j}} \sinh q_{j}=\sum_{j=1}^{k} \frac{2 \pi n_{j}}{\beta},
$$

where the sets $\left\{q_{j}\right\}$ are the sets $s_{l}^{(k)}$ for the choice $\epsilon_{1}=\cdots=\epsilon_{k}=+$, and are are in one-toone correspondence with the possible configurations of numbers $\left\{n_{1}, \ldots, n_{k}\right\}$ (there may be ambiguities in this correspondence). The values $\sum_{j=1}^{k} \frac{2 \pi n_{j}}{\beta}$ must be in $\mathbb{Z}$ or in $\mathbb{Z}+\frac{1}{2}$ (or, in general, $\mathbb{Z}+\omega)$, in order to implement the correct quasi-periodicity conditions; this is where our finite-temperature form factor expansion (4.6) is made to agree with the KMS identity.

- The quantities $\tilde{F}_{\alpha_{1}, \ldots, \alpha_{k}}^{\mathcal{O}_{1}}\left(\theta_{1}, \ldots, \theta_{k} ; \beta\right)$ for $\theta_{j}=q_{j}+i \pi / 2$ and for $\epsilon_{1}=\cdots=\epsilon_{k}=+$, times the square root of the residue of $\rho_{\alpha_{1}, \ldots, \alpha_{k}}\left(\theta_{1}, \ldots, \theta_{k}\right)$ at these same values, are proportional to the matrix elements ${ }_{\beta}\left\langle\operatorname{vac}|\mathcal{O}| n_{1}, \ldots, n_{k}\right\rangle_{\beta}$.

Note that for the case of one particle, one should recover the energy spectrum $E_{n, a}=$ $m_{a} \cosh q_{n}$ with $m_{a} \sinh q_{n}=\frac{2 \pi n}{\beta}$ and $n \in \mathbb{Z}$ if $f_{a}=0, n \in \mathbb{Z}+\frac{1}{2}$ if $f_{a}=1$. This indicates that

$$
\rho_{\alpha}(\theta)=\frac{1}{1-(-1)^{f_{a}} e^{-\epsilon \beta m_{a} \cosh \theta}}
$$

and that $\tilde{F}_{\alpha}(\theta)=F_{\alpha}(\theta)$.

Note also that in the free Majorana theory, one simply has

$$
\rho_{\alpha_{1}, \ldots, \alpha_{k}}\left(\theta_{1}, \ldots, \theta_{k}\right)=\frac{1}{\prod_{j=1}^{k}\left(1+e^{-\epsilon_{j} \beta m \cosh \theta_{j}}\right)}
$$

with $\tilde{F}=F$ for all excitation numbers, and this indeed reproduces the right energy levels in the quantization on the circle as well as the correct matrix elements [2]. In fact, for any free theory we have $\tilde{F}=F$.

It is now possible to understand the prescription (4.8) for deforming the integration contours in order to avoid possible kinematic poles in the finite-temperature form factors. Indeed, by the principles above, the finite-temperature form factor expansion is really an analytical continuation of the sum representation of two-point functions coming from the quantization on the circle. Hence, it is natural that integration contours be defined in the complex plane to avoid kinematic poles, and the direction of the deformation is exactly the one giving the proper correspondence between the expansions (4.6) and (3.4).

It is also possible to understand the restrictions on the directions of the cut of semi-local operators, as explained in the second comment after (4.6). Indeed, the finite-temperature form factor $F_{\alpha_{1}, \ldots, \alpha_{k}}^{\mathcal{O}_{1}}\left(\theta_{1}, \ldots, \theta_{k} ; \beta\right)$ is, in a sense, the analytical continuation of the matrix element $\left\langle\operatorname{vac}\left|\mathcal{O}_{1}\right| n_{1}, \ldots, n_{k}\right\rangle_{\beta}$ in the quantization on the circle, which describes "one half" of the twopoint function. In a path integral formulation, this matrix element corresponds to a path integral on the half-cylinder, say, $\tau>0, \mathrm{x} \in[0, \beta], \beta \equiv 0$ with some boundary condition at $\tau=0$ (the excited state) and some asymptotic condition at $\tau \rightarrow \infty$ (the vacuum). But since by construction the function $F_{\alpha_{1}, \ldots, \alpha_{k}}^{\mathcal{O}_{1}}\left(\theta_{1}, \ldots, \theta_{k} ; \beta\right)$ has no "knowledge" of the other operator $\mathcal{O}_{2}$ of the two-point function, it always "stand" in the natural sector given by the trace, even if this sector is modified by the cut emanating from $\mathcal{O}_{2}$ in the actual correlation function. Hence, it is important that the cut associated to $\mathcal{O}_{2}$ does not change this sector, that is, that it does not affect the conditions at $\tau \rightarrow \infty$ neither those in the region of $\tau$ present between the position of 
$\mathcal{O}_{1}$ and that of $\mathcal{O}_{2}$. Similar arguments apply to the function $F_{-\alpha_{k}, \ldots,-\alpha_{1}}^{\mathcal{O}_{2}}\left(\theta_{k}, \ldots, \theta_{1} ; \beta\right)$, and this shows that the cut of $\mathcal{O}_{1}$ must be towards the right, and that of $\mathcal{O}_{2}$, towards the left, when both are operators associated to twist fields.

To be more precise, if the cut associated to $\mathcal{O}_{2}$ does affect the sector in which $\mathcal{O}_{1}$ stands, then the only way to provide this information is by modifying the choice of the discrete values of rapidities in $F_{\alpha_{1}, \ldots, \alpha_{k}}^{\mathcal{O}_{1}}\left(\theta_{1}, \ldots, \theta_{k} ; \beta\right)$ that will form the states on the circle; that is, to modify the analytic structure of the measure $\rho$. This is indeed what is expected to happen: finitetemperature form factors of twist fields should have an analytical structure that provides the appropriate shift of the poles of the measure in order to produce the change of sector. Then, we actually expect this to be enough information for the finite-temperature form factor of $\mathcal{O}_{1}$ to be in this different sector whenever $\mathcal{O}_{1}$ is local with respect to the fundamental fields corresponding to the particles involved. In that case, the cut of $\mathcal{O}_{2}$ can indeed be in any direction, the expansion (4.6) will stay valid. Otherwise, if $\mathcal{O}_{1}$ is itself semi-local with respect to the fundamental fields, then a change of the analytic structure of the measure is not enough, hence the cut of $\mathcal{O}_{2}$ must be in opposite direction. This phenomenon is indeed what is observed in the Majorana theory.

We can circumvent the restriction on directions of the cuts of the twist fields by "twisting" the construction; this is done in the next section.

\section{Twisted constructions}

\subsection{The twisted inner product}

The construction of the previous section can be fruitfully modified when there is a $U(1)$ invariance (or sub-group thereof) in the theory, by changing the quasi-periodicity properties of the fundamental fermion field in imaginary time. We still consider the space $\mathcal{L}$ of endomorphisms of $\mathcal{H}$, but now as a Hilbert space with a different inner product structure:

$$
(A, B)_{\omega}=\frac{\operatorname{Tr}\left(e^{-\beta H+2 \pi i \omega Q} A^{\dagger} B\right)}{\operatorname{Tr}\left(e^{-\beta H+2 \pi i \omega Q}\right)} \equiv\left\langle\left\langle A^{\dagger} B\right\rangle\right\rangle_{\beta}^{\omega},
$$

where $Q$ is the Hermitian conserved charge associated to the $U(1)$ symmetry. Then we again have

$$
(A, B)_{\omega}^{*}=(B, A)_{\omega} .
$$

Now, we can still consider, in order to have a basis, the set of operators (4.2). We have

$$
Z_{\alpha}(\theta) e^{2 \pi i \omega Q}=e^{-2 \pi i \omega q(\alpha)} e^{2 \pi i \omega Q} Z_{\alpha}(\theta)
$$

where $q(\alpha)$ is the charge ${ }^{2}$ of the excitation $\alpha$. Again with diagonal scattering, using this it is possible to write down all inner products (5.1) of the operator (4.2) in a simple way:

$$
\begin{aligned}
& \left(D_{\alpha_{1}, \ldots, \alpha_{k}}\left(\theta_{1}, \ldots, \theta_{k}\right), D_{\alpha_{1}^{\prime}, \ldots, \alpha_{l}^{\prime}}\left(\theta_{1}^{\prime}, \ldots, \theta_{l}^{\prime}\right)\right)_{\omega} \\
& \quad=\delta_{k, l} \prod_{i=1}^{k} \frac{\epsilon_{i}^{1-f_{a_{i}}} \delta_{a_{i}, a_{i}^{\prime}} \delta\left(\theta_{i}-\theta_{i}^{\prime}\right)}{1-(-1)^{f_{a_{i}}} e^{2 \pi i \omega q\left(\alpha_{i}\right)} e^{-\epsilon_{i} \beta m_{a_{i}} \cosh \theta_{i}}} .
\end{aligned}
$$

Here, we have assumed the ordering (4.1) for both members of the inner product.

\footnotetext{
${ }^{2}$ In particular the charge of the fundamental fermion, with $\epsilon=+$, is 1 , and in general we have $q(a,+)=$ $-q(a,-)$.
} 
Note that simple "crossing" relations hold again for operators in $\operatorname{End}_{R L}(\mathcal{L})$ :

$$
\begin{aligned}
& \left(D_{\alpha_{1}, \ldots, \alpha_{k}}\left(\theta_{1}, \ldots, \theta_{k}\right), \phi_{L}(A) D_{\alpha_{1}^{\prime}, \ldots, \alpha_{l}^{\prime}}\left(\theta_{1}^{\prime}, \ldots, \theta_{l}^{\prime}\right)\right)=e^{-2 \pi i \omega q\left(\alpha_{l}^{\prime}\right)} e^{\epsilon_{l}^{\prime} \beta m_{a_{l}^{\prime}} \cosh \theta_{l}^{\prime}} \\
& \quad \times\left(D_{\alpha_{1}, \ldots, \alpha_{k}, \bar{\alpha}_{l}^{\prime}}\left(\theta_{1}, \ldots, \theta_{k}, \theta_{l}^{\prime}\right), \phi_{L}(A) D_{\alpha_{1}^{\prime}, \ldots, \alpha_{l-1}^{\prime}}\left(\theta_{1}^{\prime}, \ldots, \theta_{l-1}^{\prime}\right)\right) \quad\left(\theta_{l}^{\prime} \neq \theta_{i} \forall i\right)
\end{aligned}
$$

and

$$
\begin{aligned}
& \left(D_{\alpha_{1}, \ldots, \alpha_{k}}\left(\theta_{1}, \ldots, \theta_{k}\right), \phi_{R}(A) D_{\alpha_{1}^{\prime}, \ldots, \alpha_{l}^{\prime}}\left(\theta_{1}^{\prime}, \ldots, \theta_{l}^{\prime}\right)\right) \\
& \quad=\left(D_{\bar{\alpha}_{1}^{\prime}, \alpha_{1}, \ldots, \alpha_{k}}\left(\theta_{1}^{\prime}, \theta_{1}, \ldots, \theta_{k}\right), \phi_{R}(A) D_{\alpha_{2}^{\prime}, \ldots, \alpha_{l}^{\prime}}\left(\theta_{2}^{\prime}, \ldots, \theta_{l}^{\prime}\right)\right) \quad\left(\theta_{1}^{\prime} \neq \theta_{i} \forall i\right) .
\end{aligned}
$$

\subsection{Twisted finite-temperature form factor expansion}

Inverting (5.2), we now have the twisted finite-temperature form factor expansion (as before, we assume that $x>0)$ :

$$
\begin{aligned}
& \left\langle\left\langle\mathcal{O}_{1}(x, t) \mathcal{O}_{2}(0,0)\right\rangle\right\rangle_{\beta}^{\omega} \\
& =e^{\Delta \mathcal{E} x} \sum_{k=0}^{\infty} \sum_{\alpha_{1}, \ldots, \alpha_{k}} \int_{\left\{\operatorname{Im}\left(\theta_{j}\right)=\epsilon_{j} 0^{+}\right\}} \frac{d \theta_{1} \cdots d \theta_{k} \prod_{j=1}^{k} \epsilon_{j}^{1-f_{a_{j}}} e^{\sum_{j=1}^{k} \epsilon_{j}\left(i m_{a_{j}} x \sinh \theta_{j}-i m_{a_{j}} t \cosh \theta_{j}\right)}}{k ! \prod_{j=1}^{k}\left(1-(-1)^{f_{a_{j}}} e^{2 \pi i \omega q\left(\alpha_{j}\right)} e^{-\epsilon_{j} \beta m_{a_{j}} \cosh \theta_{j}}\right)} \\
& \quad \times{ }^{\omega} F_{\alpha_{1}, \ldots, \alpha_{k}}^{\mathcal{O}_{1}}\left(\theta_{1}, \ldots, \theta_{k} ; \beta\right){ }^{\omega} F_{-\alpha_{k}, \ldots,-\alpha_{1}}^{\mathcal{O}_{2}}\left(\theta_{k}, \ldots, \theta_{1} ; \beta\right),
\end{aligned}
$$

where we have defined twisted finite-temperature form factors as the normalised matrix elements $^{3}$ :

$$
\begin{aligned}
& { }^{\omega} F_{\alpha_{1}, \ldots, \alpha_{n}}^{\mathcal{O}}\left(\theta_{1}, \ldots, \theta_{n} ; \beta\right) \\
& =\prod_{i=1}^{n}\left[\epsilon_{i}^{1-f_{a_{i}}}\left(1-(-1)^{f_{a_{i}}} e^{2 \pi i \omega q\left(\alpha_{i}\right)} e^{-\epsilon_{i} \beta m_{a_{i}} \cosh \theta_{i}}\right)\right]\left(\mathcal{O}^{\dagger}(0,0), D_{\alpha_{1}, \ldots, \alpha_{n}}\left(\theta_{1}, \ldots, \theta_{n}\right)\right)_{\omega} .
\end{aligned}
$$

and used the identity

$$
\left({ }^{\omega} F_{\alpha_{1}, \ldots, \alpha_{k}}^{\mathcal{O}_{2}^{\dagger}}\left(\theta_{1}^{*}, \ldots, \theta_{k}^{*} ; \beta\right)\right)^{*}={ }^{\omega} F_{-\alpha_{k}, \ldots,-\alpha_{1}}^{\mathcal{O}_{2}}\left(\theta_{k}, \ldots, \theta_{1} ; \beta\right)
$$

which essentially follows from (6.2) below. Also, we have symmetrised over the orderings of rapidities. The quantity $\Delta \mathcal{E}$ is non-zero whenever $\mathcal{O}_{1}$ is a twist field $\sigma_{\eta_{1}}^{\omega_{1}}$, and is now given by

$$
\Delta \mathcal{E}= \begin{cases}\mathcal{E}\left[\frac{1}{2}+\omega+\omega_{1}\right]-\mathcal{E}\left[\frac{1}{2}+\omega\right] & \left(\eta_{1}=+\right), \\ \mathcal{E}\left[\frac{1}{2}+\omega\right]-\mathcal{E}\left[\frac{1}{2}+\omega-\omega_{1}\right] & \left(\eta_{1}=-\right),\end{cases}
$$

where $\mathcal{E}[\nu]$ is the energy of the vacuum $\left|\operatorname{vac}_{\nu}\right\rangle_{\beta}$ (see the discussion around (3.9)). Again, when both $\mathcal{O}_{1}$ and $\mathcal{O}_{2}$ are semi-local with respect to the fundamental fermion field, the finitetemperature form factor expansion (4.6) is valid only when the cut of $\mathcal{O}_{1}$ extends towards the right (positive $x$ direction) and that of $\mathcal{O}_{2}$ extends towards the left (negative $x$ direction). This is justified in the same way as before, through the relation between (5.5) and a form factor expansion on the circle (3.4). Note that with this prescription on the directions of the cuts, one now produces the correlation functions

$$
\beta\left\langle\operatorname{vac}_{\frac{1}{2}+\omega+\omega_{1}}\left|\hat{\mathcal{O}}_{1}(\mathrm{x}, \tau) \hat{\mathcal{O}}_{2}(0,0)\right| \operatorname{vac}_{\frac{1}{2}+\omega-\omega_{2}}\right\rangle_{\beta}
$$

with $x=\tau$ and $t=i \mathrm{x}$. With $\omega_{1}=-\omega_{2}$, it is now possible to have the NS vacuum by choosing $\omega=-\omega_{1}$.

\footnotetext{
${ }^{3}$ Again, note that the function $F_{\alpha_{1}, \ldots, \alpha_{n}}^{\mathcal{O}}\left(\theta_{1}, \ldots, \theta_{n} ; \beta\right)$ has no delta-function contributions at colliding rapidities.
} 


\section{Formal structure and a generalisation of CFT's mapping to the cylinder}

\subsection{The space $\mathcal{L}$ as a Fock space, and physical interpretation}

We have seen how the space $\mathcal{L}$ of operators on $\mathcal{H}$ can be used to obtain infinite series expressions of correlation functions (4.6) and (5.5). The important objects are the finite-temperature form factors (4.7), or the twisted version $(5.6)$, which are certain matrix elements on the space $\mathcal{L}$. It will be convenient, here, to introduce the normalised operators

$$
d_{\alpha_{1}, \ldots, \alpha_{k}}\left(\theta_{1}, \ldots, \theta_{k}\right)=\prod_{i=1}^{k} \frac{1}{g_{\alpha_{i}}\left(\theta_{i}\right)} D_{\alpha_{1}, \ldots, \alpha_{k}}\left(\theta_{1}, \ldots, \theta_{k}\right)
$$

with, in the general twisted case,

$$
g_{\alpha}(\theta)=\frac{\epsilon^{1-f_{a}}}{1-(-1)^{f_{a}} e^{2 \pi i \omega q(\alpha)} e^{-\epsilon \beta m_{a} \cosh \theta}} .
$$

Then, we have

$$
F_{\alpha_{1}, \ldots, \alpha_{k}}^{\mathcal{O}}\left(\theta_{1}, \ldots, \theta_{k} ; \beta\right)=\left(\mathcal{O}^{\dagger}(0,0), d_{\alpha_{1}, \ldots, \alpha_{k}}\left(\theta_{1}, \ldots, \theta_{k}\right)\right)
$$

and the "crossing" relations (4.4), (4.5) change into

$$
\begin{aligned}
& \left(d_{\alpha_{1}, \ldots, \alpha_{k}}\left(\theta_{1}, \ldots, \theta_{k}\right), \phi_{L}(A) d_{\alpha_{1}^{\prime}, \ldots, \alpha_{l}^{\prime}}\left(\theta_{1}^{\prime}, \ldots, \theta_{l}^{\prime}\right)\right)_{\omega} \\
& \quad=\left(d_{\alpha_{1}, \ldots, \alpha_{k}, \bar{\alpha}_{l}^{\prime}}\left(\theta_{1}, \ldots, \theta_{k}, \theta_{l}^{\prime}\right), \phi_{L}(A) d_{\alpha_{1}^{\prime}, \ldots, \alpha_{l-1}^{\prime}}\left(\theta_{1}^{\prime}, \ldots, \theta_{l-1}^{\prime}\right)\right)_{\omega} \quad\left(\theta_{l}^{\prime} \neq \theta_{i} \forall i\right)
\end{aligned}
$$

and

$$
\begin{aligned}
& \left(d_{\alpha_{1}, \ldots, \alpha_{k}}\left(\theta_{1}, \ldots, \theta_{k}\right), \phi_{R}(A) d_{\alpha_{1}^{\prime}, \ldots, \alpha_{l}^{\prime}}\left(\theta_{1}^{\prime}, \ldots, \theta_{l}^{\prime}\right)\right)_{\omega}=e^{2 \pi i \omega q\left(\alpha_{1}^{\prime}\right)} e^{-\epsilon_{1}^{\prime} \beta m_{a_{1}^{\prime}} \cosh \theta_{1}^{\prime}} \\
& \quad \times\left(d_{\bar{\alpha}_{1}^{\prime}, \alpha_{1}, \ldots, \alpha_{k}}\left(\theta_{1}^{\prime}, \theta_{1}, \ldots, \theta_{k}\right), \phi_{R}(A) d_{\alpha_{2}^{\prime}, \ldots, \alpha_{l}^{\prime}}\left(\theta_{2}^{\prime}, \ldots, \theta_{l}^{\prime}\right)\right)_{\omega} \quad\left(\theta_{1}^{\prime} \neq \theta_{i} \forall i\right) .
\end{aligned}
$$

In order to describe in a convenient way the space spanned by $d_{\alpha_{1}, \ldots, \alpha_{k}}\left(\theta_{1}, \ldots, \theta_{k}\right)$, we introduce the following operators acting on $\mathcal{L}$ :

$$
\mathcal{Z}_{\alpha}^{\dagger}(\theta), \mathcal{Z}_{\alpha}(\theta) \in \operatorname{End}(\mathcal{L})
$$

with the following properties:

$$
\mathcal{Z}_{\alpha}^{\dagger}(\theta) \mathbf{1}_{\mathcal{H}}=d_{\alpha}(\theta), \quad \mathcal{Z}_{\alpha_{1}}^{\dagger}\left(\theta_{1}\right) d_{\alpha_{2}, \ldots, \alpha_{k}}\left(\theta_{2}, \ldots, \theta_{k}\right)=d_{\alpha_{1}, \ldots, \alpha_{k}}\left(\theta_{1}, \ldots, \theta_{k}\right), \quad \mathcal{Z}_{\alpha}(\theta) \mathbf{1}_{\mathcal{H}}=0
$$

and satisfying the following exchange relations:

$$
\begin{aligned}
& \mathcal{Z}_{\alpha_{1}}\left(\theta_{1}\right) \mathcal{Z}_{\alpha_{2}}\left(\theta_{2}\right)-S_{\alpha_{1}, \alpha_{2}}\left(\theta_{1}-\theta_{2}\right) \mathcal{Z}_{\alpha_{2}}\left(\theta_{2}\right) \mathcal{Z}_{\alpha_{1}}\left(\theta_{1}\right)=0 \\
& \mathcal{Z}_{\alpha_{1}}^{\dagger}\left(\theta_{1}\right) \mathcal{Z}_{\alpha_{2}}^{\dagger}\left(\theta_{2}\right)-S_{\alpha_{1}, \alpha_{2}}\left(\theta_{1}-\theta_{2}\right) \mathcal{Z}_{\alpha_{2}}^{\dagger}\left(\theta_{2}\right) \mathcal{Z}_{\alpha_{1}}^{\dagger}\left(\theta_{1}\right)=0 \\
& \mathcal{Z}_{\alpha_{1}}\left(\theta_{1}\right) \mathcal{Z}_{\alpha_{2}}^{\dagger}\left(\theta_{2}\right)-S_{\alpha_{2}, \alpha_{1}}\left(\theta_{2}-\theta_{1}\right) \mathcal{Z}_{\alpha_{2}}^{\dagger}\left(\theta_{2}\right) \mathcal{Z}_{\alpha_{1}}\left(\theta_{1}\right)=\frac{1}{g_{\alpha_{1}}\left(\theta_{1}\right)} \delta_{\alpha_{1}, \alpha_{2}} \delta\left(\theta_{1}-\theta_{2}\right),
\end{aligned}
$$

where $\delta_{\alpha_{1}, \alpha_{2}}=\delta_{a_{1}, a_{2}} \delta_{\epsilon_{1}, \epsilon_{2}}$ and where

$$
S_{\alpha_{1}, \alpha_{2}}(\theta)= \begin{cases}S_{a_{1}, a_{2}}(\theta) & \left(\epsilon_{1}=\epsilon_{2}\right) \\ S_{a_{2}, a_{1}}(-\theta) & \left(\epsilon_{1}=-\epsilon_{2}\right)\end{cases}
$$


The space $\mathcal{H}$ is then seen as a Fock space over the algebra (6.4), with vacuum vector $\mathbf{1}_{\mathcal{H}}$ annihilated by $\mathcal{Z}_{\alpha}(\theta)$. The algebra (6.4) has exactly the structure of the Zamolodchikov-Faddeev algebra (2.1) with diagonal scattering, but with twice as many particles. The physical interpretation is that the "states" $d_{\alpha_{1}, \ldots, \alpha_{k}}\left(\theta_{1}, \ldots, \theta_{k}\right)$ correspond to configurations of stable "additional particles" $(\epsilon=+)$ and "missing particles" or holes $(\epsilon=-)$ in a thermal bath (we will call both "excitations"), both created by the operators $\mathcal{Z}_{\alpha}^{\dagger}(\theta)$, and scattering through the matrix $S_{\alpha_{1}, \alpha_{2}}\left(\theta_{1}-\theta_{2}\right)$. They are stable, since the states with $n$ excitations have no overlap with those with $n^{\prime} \neq n$ excitations. It is both this stability and the fact that matrix elements should have nice analytical properties that suggests that the correspondence between expansions in the quantization on the circle and finite-temperature form factor expansions holds individually for every term with a given excitation number. Only in integrable quantum field theory can we expect these two properties together.

In order to have a better picture of the "particle" and "hole" states that we are discussing, one should recall that we defined the $i n$-states of $\mathcal{H}$ by multiple action of $\bar{Z}_{a}(\theta)$ on $\mid$ vac $\rangle$ with ordered rapidities, decreasing from left to right. When this order is not satisfied, one has an "intermediate" state, which is a useful concept only in integrable quantum field theory. It corresponds to having wave packets ordered such that some interact in the far past (like for out states), while others interact in the far future (like for in states). Essentially, the order of the operators $\bar{Z}_{a}(\theta)$ acting on $|\mathrm{vac}\rangle$ corresponds to the order of the wave packets themselves along the $x$ axis, when time is taken to go "upward" (in the positive $y$ direction). When we put an operator $\bar{Z}_{a}(\theta)$ inside the finite-temperature trace (evaluating the trace in the in basis, for instance), we take situations with various numbers of in particles at various ordered rapidities, and put an additional wave packet far to the left, generically producing "intermediate" states. This is the sense in which the operator $\bar{Z}_{a}(\theta)$ corresponds to an additional particle in a thermal bath. Similarly, $Z_{a}(\theta)$ is taking away a particle from the thermal bath, by first bringing its wave packet far to the left. Recall that it is because we keep the order of the wave packets fixed while varying the rapidities that matrix elements of operators on $\mathcal{H}$ in "intermediate" states are meromorphic functions. With the previous discussion, this lends support to the fact that the basis $D_{\alpha_{1}, \ldots, \alpha_{k}}\left(\theta_{1}, \ldots, \theta_{k}\right)$ of $\mathcal{L}$ should produce matrix elements with nice analytical properties.

It is also interesting to note a nice physical interpretation for the expected kinematical poles in the finite-temperature form factors, occurring at colliding rapidities when they are associated to opposite signs of $\epsilon$. These poles can be seen, in the finite-temperature form factor expansion, to lead to powers of the time variable $t$, instead of exponential factors. This corresponds to the fact that a particle and a hole can annihilate and re-form themselves at arbitrary large time differences without cost in energy. For a given excitation number, various powers in $t$ will occur, which can more or less be made in correspondence with various simultaneous annihilating and re-creating of particle-hole pairs. It it these processes that make the computation of large-time dynamics from finite-temperature form factors difficult: a re-summation of these powers of $t$ is necessary.

The fact that the Hilbert space $\mathcal{L}$ is the same as an ordinary Hilbert space with twice as many "particles" does not mean that the finite-temperature theory is the same as a zero-temperature one with such particles. Indeed, another ingredient that defines a theory is the set of local operators (in particular, the energy density), and this set looks very different on a finite-temperature Hilbert space. In fact, it would be very interesting to study the structure of the energy density on $\mathcal{L}$.

\subsection{A tool for evaluating traces and mapping to the cylinder}

This is again a generalisation of a concept introduced in [2]. It is possible to construct the left action of $\bar{Z}_{\alpha}$ through the operators $\mathcal{Z}_{\alpha}^{\dagger}$ and $\mathcal{Z}_{\bar{\alpha}}$ (the right action is more complicated, from the 
definition of the operators $\mathcal{Z}_{\alpha}^{\dagger}$ and $\mathcal{Z}_{\alpha}$, but it is sufficient to consider the left action). Indeed, one can verify that the mapping

$$
\phi_{L}\left(\bar{Z}_{\alpha}(\theta)\right)=\mathcal{Z}_{\alpha}^{\dagger}(\theta) g_{\alpha}(\theta)+\mathcal{Z}_{\bar{\alpha}}(\theta) g_{\bar{\alpha}}(\theta) \quad(\bar{\alpha}=(a,-\epsilon) \text { for } \alpha=(a, \epsilon))
$$

is an isomorphism of the algebra satisfied by $\bar{Z}_{\alpha}(\theta)$ :

$$
\bar{Z}_{\alpha_{1}}\left(\theta_{1}\right) \bar{Z}_{\alpha_{2}}\left(\theta_{2}\right)-S_{\alpha_{1}, \alpha_{2}}\left(\theta_{1}-\theta_{2}\right) \bar{Z}_{\alpha_{2}}\left(\theta_{2}\right) \bar{Z}_{\alpha_{1}}\left(\theta_{1}\right)=\epsilon_{2}^{1-f_{a_{2}}} \delta_{\epsilon_{1},-\epsilon_{2}} \delta_{a_{1}, a_{2}} \delta\left(\theta_{1}-\theta_{2}\right),
$$

using the property $S_{\bar{\alpha}_{2}, \alpha_{1}}\left(\theta_{2}-\theta_{1}\right)=S_{\alpha_{1}, \alpha_{2}}\left(\theta_{1}-\theta_{2}\right)$ as well as $g_{\alpha}(\theta)+(-1)^{1-f_{a}} g_{\bar{\alpha}}(\theta)=\epsilon^{1-f_{a}}$. From this one can conclude the equality

$$
\left(\mathbf{1}_{\mathcal{H}}, \phi_{L}\left(\bar{Z}_{\alpha_{1}}\left(\theta_{1}\right)\right) \cdots \phi_{L}\left(\bar{Z}_{\alpha_{k}}\left(\theta_{k}\right)\right) \mathbf{1}_{\mathcal{H}}\right)=\left(\mathbf{1}_{\mathcal{H}}, \bar{Z}_{\alpha_{1}}\left(\theta_{1}\right) \cdots \bar{Z}_{\alpha_{k}}\left(\theta_{k}\right)\right)
$$

by bringing on both sides, for instance, all factors with $\epsilon=+$ to the right, by evaluating the resulting right-hand side explicitly using the inner product (5.2), and by evaluating the lefthand side using the representation (6.5). Then, restrict all rapidities numbered $j+1, \ldots, k$ to be different from one another, and restrict all rapidities numbered $1, \ldots, j-1$ to be different from one another. The left-hand side evaluates to

$$
\left(\bar{Z}_{\alpha_{j-1}}\left(\theta_{j-1}\right) \cdots \bar{Z}_{\alpha_{1}}\left(\theta_{1}\right), \phi_{L}\left(\bar{Z}_{\alpha_{j}}\left(\theta_{j}\right)\right) \bar{Z}_{\alpha_{j+1}}\left(\theta_{j+1}\right) \cdots \bar{Z}_{\alpha_{k}}\left(\theta_{k}\right)\right)
$$

whereas the right-hand side evaluates to

$$
\left(\bar{Z}_{\alpha_{j-1}}\left(\theta_{j-1}\right) \cdots \bar{Z}_{\alpha_{1}}\left(\theta_{1}\right), \bar{Z}_{\alpha_{j}}\left(\theta_{j}\right) \bar{Z}_{\alpha_{j+1}}\left(\theta_{j+1}\right) \cdots \bar{Z}_{\alpha_{k}}\left(\theta_{k}\right)\right) .
$$

The equality of these two expressions shows the equality for all matrix elements, hence shows (6.5).

From relation (6.5), one can write explicitly products $\bar{Z}_{\alpha_{1}}\left(\theta_{1}\right) \cdots \bar{Z}_{\alpha_{k}}\left(\theta_{k}\right)$ as linear combinations of the basis elements $d_{\alpha_{1}^{\prime}, \ldots, \alpha_{l}^{\prime}}\left(\theta_{1}^{\prime}, \ldots, \theta_{l}^{\prime}\right)$ (for $l \leq k$ and where primed variables form a subset of un-primed variables), using

$$
\bar{Z}_{\alpha_{1}}\left(\theta_{1}\right) \cdots \bar{Z}_{\alpha_{k}}\left(\theta_{k}\right)=\phi_{L}\left(\bar{Z}_{\alpha_{1}}\left(\theta_{1}\right)\right) \cdots \phi_{L}\left(\bar{Z}_{\alpha_{k}}\left(\theta_{k}\right)\right) \mathbf{1}_{\mathcal{H}}
$$

In particular, using cyclic properties of the trace, one can relax the restriction of having different rapidities in (6.2), and one obtains

$$
\begin{aligned}
& \left(d_{\alpha_{1}^{\prime}, \ldots, \alpha_{l}^{\prime}}\left(\theta_{1}^{\prime}, \ldots, \theta_{l}^{\prime}\right), \phi_{L}(A) d_{\alpha_{1}, \ldots, \alpha_{k}}\left(\theta_{1}, \ldots, \theta_{k}\right)\right) \\
& \quad=\left(d_{\alpha_{1}^{\prime}, \ldots, \alpha_{l-1}^{\prime}}\left(\theta_{1}^{\prime}, \ldots, \theta_{l-1}^{\prime}\right), \phi_{L}(A) d_{\alpha_{1}, \ldots, \alpha_{k}, \alpha_{l}^{\prime}}\left(\theta_{1}, \ldots, \theta_{k}, \theta_{l}^{\prime}\right)\right) \\
& \quad+\sum_{j=1}^{k} S_{\alpha_{j}, \alpha_{j+1}}\left(\theta_{j}-\theta_{j+1}\right) \cdots S_{\alpha_{j}, \alpha_{k}}\left(\theta_{j}, \theta_{k}\right) \frac{\delta_{\alpha_{j}, \alpha_{l}^{\prime}} \delta\left(\theta_{j}-\theta_{l}^{\prime}\right)}{g_{\alpha_{j}}\left(\theta_{j}\right)} \\
& \quad \times\left(d_{\alpha_{1}^{\prime}, \ldots, \alpha_{l-1}^{\prime}}\left(\theta_{1}^{\prime}, \ldots, \theta_{l-1}^{\prime}\right), \phi_{L}(A) d_{\alpha_{1}, \ldots, \hat{\alpha}_{j}, \ldots, \alpha_{k}}\left(\theta_{1}, \ldots, \hat{\theta}_{j}, \ldots, \theta_{k}\right)\right),
\end{aligned}
$$

where the hat means that the variable is missing.

It is now possible to generalise, in some sense, the concept of "mapping to the cylinder" that can be used in conformal field theory in order to evaluate finite-temperature correlation functions. In conformal field theory, one has a mapping (of vertex operator algebras) $\mathcal{O} \mapsto \hat{\mathcal{O}}$ such that correlation functions of $\hat{\mathcal{O}}$ on the cylinder are equal to correlation functions of $\mathcal{O}$ on the plane (for instance, for the stress energy tensor one has $T \mapsto\left(\frac{\beta}{2 \pi} T+\frac{c}{24}\right) z^{-2}$ where $w$ is the coordinate on the cylinder, $z=e^{2 \pi w / \beta}$ is the coordinate on the plane and $c$ is the central 
charge). We can generalise this here at the level of form factors. We seek a linear map $\Omega$ from $\mathcal{L}$ to $\mathcal{L}$ such that

$$
\begin{array}{r}
\left(\mathbf{1}_{\mathcal{H}}, \phi_{L}(\Omega A) d_{\left(a_{1},+\right), \ldots,\left(a_{k},+\right),\left(a_{l}^{\prime},-\right), \ldots,\left(a_{1}^{\prime},-\right)}\left(\theta_{1}, \ldots, \theta_{k}, \theta_{l}^{\prime}, \ldots, \theta_{1}^{\prime}\right)\right) \\
={ }_{a_{1}^{\prime}, \ldots, a_{l}^{\prime}}\left\langle\theta_{1}^{\prime}, \ldots, \theta_{l}^{\prime}|A| \theta_{1}, \ldots, \theta_{k}\right\rangle_{a_{1}, \ldots, a_{k}} \quad\left(\theta_{i}^{\prime} \neq \theta_{j} \forall i, j\right),
\end{array}
$$

where $A \in \mathcal{L}$. This requirement is inspired by the fact that

$$
\begin{gathered}
\lim _{\beta \rightarrow \infty}\left(\mathbf{1}_{\mathcal{H}}, \phi_{L}(A) d_{\left(a_{1},+\right), \ldots,\left(a_{k},+\right),\left(a_{l}^{\prime},-\right), \ldots,\left(a_{1}^{\prime},-\right)}\left(\theta_{1}, \ldots, \theta_{k}, \theta_{l}^{\prime}, \ldots, \theta_{1}^{\prime}\right)\right) \\
=a_{1}^{\prime}, \ldots, a_{l}^{\prime}\left\langle\theta_{1}^{\prime}, \ldots, \theta_{l}^{\prime}|A| \theta_{1}, \ldots, \theta_{k}\right\rangle_{a_{1}, \ldots, a_{k}} \quad\left(\theta_{i}^{\prime} \neq \theta_{j} \forall i, j\right)
\end{gathered}
$$

thanks to (6.2). Note that this in fact completely fixes the map $\phi_{L} \circ \Omega$, thanks to (6.6), and by injection the map $\Omega$, if it exists.

In order to describe the map $\Omega$, it is convenient to consider elements of $\mathcal{L}$ that have simple expectation values on $\mathcal{H}$. We consider products where operators $\bar{Z}_{\alpha}(\theta)$ are normal-ordered with respect to the vacuum in $\mathcal{H}$ : operators $Z^{a}(\theta)$ are placed to the right of all operators $\bar{Z}_{a}(\theta)$, without taking any delta-function term (but taking all $S$-matrices involved in the exchanges). We will denote the normal-ordering of $A$ by the standard :A:. The set of all normal-ordered operators spans $\mathcal{L}$. On the other hand, there is a natural normal-ordering with respect to the vacuum $\mathbf{1}_{\mathcal{H}}$ in $\mathcal{L}$ : it is the one whereby operators $\mathcal{Z}$ are placed to the right of operators $\mathcal{Z}^{\dagger}$. We will denote the normal-ordering of $\mathcal{A} \in \operatorname{End}(\mathcal{L})$ by ${ }_{*}^{*} \mathcal{A}_{*}^{*}$. Then, it is simple to see that for all normal-ordered $A$ (that is, $: A:=A$ ),

$$
{ }_{*}^{*} \phi_{L}(A)_{*}^{*}
$$

can be put in place of $\phi_{L}(\Omega(A))$ in (6.7) in order to have equality. Indeed if $A$ contains exactly $l$ operators of type $\bar{Z}_{a}$ and $k$ operators of type $Z^{a}$, then the equality is clear; otherwise, both sides are zero, hence the equality still holds. However, this does not yet show that the map $\Omega$ exists.

Now, let us choose a basis in $\mathcal{L}$, with normal-ordered elements $A_{i}$ composed of products of finitely many operators $\bar{Z}_{\alpha}$. Certainly, the set $\phi_{L}\left(A_{i}\right)$ is not a basis in $\operatorname{End}(\mathcal{L})$. However, if we are to project from the left with $\mathbf{1}_{\mathcal{H}}$, then we do obtain a basis: $\left(\mathbf{1}_{\mathcal{H}}, \phi_{L}\left(A_{i}\right) \cdot\right)$ is a basis in the dual of $\mathcal{L}$. Similarly, the space of operators ${ }_{*}^{*} \phi_{L}\left(A_{i}\right)_{*}^{*}$ gives another basis in the dual of $\mathcal{L}$ when projected from the left with $\mathbf{1}_{\mathcal{H}}$. Hence, we have the change-of-basis relation

$$
\left(\mathbf{1}_{\mathcal{H},}{ }_{*}^{*} \phi_{L}\left(A_{i}\right)_{*}^{*} \cdot\right)=\sum_{j=0}^{k_{i}} M_{i, j}\left(\mathbf{1}_{\mathcal{H}}, \phi_{L}\left(A_{j}\right) \cdot\right),
$$

where the sum is finite. Then we find, from the discussion around (6.8) and recalling that $: A_{i}:=A_{i}$,

$$
\Omega A_{i}=\sum_{j=0}^{k_{i}} M_{i, j} A_{j} .
$$

The change of basis (6.9) could be calculated explicitly, but there is a nice way of expressing it. Suppose we can find an operator $\Upsilon$ in $\operatorname{End}(\mathcal{L})$ such that

$$
\Upsilon \mathbf{1}_{\mathcal{H}}=0, \quad\left[\Upsilon, \mathcal{Z}_{\alpha}(\theta)\right]=0, \quad\left[\Upsilon, \mathcal{Z}_{\alpha}^{\dagger}(\theta)\right] g_{\alpha}(\theta)=\mathcal{Z}_{\bar{\alpha}}(\theta) g_{(a,-)}(\theta) .
$$

Then one can verify that $e^{\Upsilon *} \phi_{L}(: A:)_{*}^{*} e^{-\Upsilon} \mathbf{1}_{\mathcal{H}}=: A$ : hence that

$$
\Omega=e^{-\Upsilon} .
$$


But using the algebra (6.4), one can see that

$$
\Upsilon=\int d \theta \sum_{a} \mathcal{Z}_{(a,-)}(\theta) \mathcal{Z}_{(a,+)}(\theta) g_{(a,-)}(\theta)
$$

has the right properties. Hence we have found an explicit expression for the map, acting on the space of operators on $\mathcal{H}$, that transform finite-temperature form factors into zero-temperature form factors. This is the generalisation of the concept of "mapping to the cylinder" in conformal field theory. Finite-temperature form factors can then be calculated using

$$
\begin{gathered}
\left(\mathbf{1}_{\mathcal{H}}, \phi_{L}(A) d_{\left(a_{1},+\right), \ldots,\left(a_{k},+\right),\left(a_{l}^{\prime},-\right), \ldots,\left(a_{1}^{\prime},-\right)}\left(\theta_{1}, \ldots, \theta_{k}, \theta_{l}^{\prime}, \ldots, \theta_{1}^{\prime}\right)\right) \\
\quad={ }_{a_{1}^{\prime}, \ldots, a_{l}^{\prime}}\left\langle\theta_{1}^{\prime}, \ldots, \theta_{l}^{\prime}\left|\left(e^{\Upsilon} A\right)\right| \theta_{1}, \ldots, \theta_{k}\right\rangle_{a_{1}, \ldots, a_{k}} .
\end{gathered}
$$

It is important to note, however, that the operator $e^{\Upsilon}$ does not act on the initial Hilbert space $\mathcal{H}$, but rather on the space of operators acting on it $\mathcal{L}$. In the quantization on the line, this is not isomorphic to $\mathcal{H}$. In conformal field theory, one usually thinks about the quantization on the circle around a fixed point (radial quantization), and by the operator-state correspondence, one then has an operator acting on the Hilbert space that performs the mapping to the cylinder.

The action of $e^{\Upsilon}$ on $A \in \mathcal{L}$ can be made more explicit using (6.10). Indeed, taking $A=$ $\bar{Z}_{\alpha_{1}}\left(\theta_{1}\right) \cdots \bar{Z}_{\alpha_{k}}\left(\theta_{k}\right)$, we can write

$$
\begin{aligned}
e^{\Upsilon}\left(\bar{Z}_{\alpha_{1}}\left(\theta_{1}\right) \cdots \bar{Z}_{\alpha_{k}}\left(\theta_{k}\right)\right) & =e^{\Upsilon} \phi_{L}\left(\bar{Z}_{\alpha_{1}}\left(\theta_{1}\right)\right) \cdots \phi_{L}\left(\bar{Z}_{\alpha_{k}}\left(\theta_{k}\right)\right) \mathbf{1}_{\mathcal{H}} \\
& =e^{\Upsilon} \phi_{L}\left(\bar{Z}_{\alpha_{1}}\left(\theta_{1}\right)\right) e^{-\Upsilon} \cdots e^{\Upsilon} \phi_{L}\left(\bar{Z}_{\alpha_{k}}\left(\theta_{k}\right)\right) e^{-\Upsilon} \mathbf{1}_{\mathcal{H}}
\end{aligned}
$$

and use

$$
\begin{aligned}
& e^{\Upsilon} \phi_{L}\left(\bar{Z}_{\alpha}(\theta)\right) e^{-\Upsilon}=\phi_{L}\left(\bar{Z}_{\alpha}(\theta)\right)+\mathcal{Z}_{\bar{\alpha}}(\theta) g_{(a,-)}(\theta), \\
& \mathcal{Z}_{\bar{\alpha}}(\theta) \phi_{L}\left(\bar{Z}_{\alpha^{\prime}}\left(\theta^{\prime}\right)\right)=S_{\alpha, \alpha^{\prime}}\left(\theta-\theta^{\prime}\right) \phi_{L}\left(\bar{Z}_{\alpha^{\prime}}\left(\theta^{\prime}\right)\right) \mathcal{Z}_{\bar{\alpha}}(\theta)+\delta_{\bar{\alpha}, \alpha^{\prime}} \delta\left(\theta-\theta^{\prime}\right)
\end{aligned}
$$

to bring all the $\mathcal{Z}_{\alpha}(\theta)$ to the right, annihilating $\mathbf{1}_{\mathcal{H}}$. This leads to a natural generalisation of Wick's theorem, whereby $e^{\Upsilon} A$ is written as $A+$ operators where more and more contractions have been performed, the contractions being given by

$$
\text { contraction of } \bar{Z}_{\alpha}(\theta) \text { with } \bar{Z}_{\alpha^{\prime}}\left(\theta^{\prime}\right)=g_{(a,-)}(\theta) \delta_{\bar{\alpha}, \alpha^{\prime}}\left(\theta-\theta^{\prime}\right) \text {. }
$$

If the $S$-matrix $S_{\alpha, \alpha^{\prime}}\left(\theta-\theta^{\prime}\right)$ is equal to \pm 1 (free models), then this gives the standard Wick's theorem, and in particular it can be applied to linear combinations of the type $\int d \theta \bar{Z}_{\alpha}(\theta) f_{\theta}$ as well. In free models certain linear combinations of this type indeed represent local fields, and this immediately leads to the phenomenon of "mixing" that was described in [2] (using slightly different arguments). Of course, local fields in interacting models and twist fields in general are not simply such linear combinations, but rather are sums of operators with more and more factors of $\bar{Z}_{\alpha}(\theta)$ (since many-particle form factors are non-zero). Hence all term will contribute to any given finite-temperature form factor, and it is a non-trivial matter to re-sum these contributions.

\section{Results in the free massive Majorana theory}

\subsection{Free massive Majorana fermions}

The free massive Majorana theory with mass $m$ can be described by the action

$$
\mathcal{A}=i \int d^{2} x\left(-\psi\left(\partial_{x}+\partial_{t}\right) \psi+\bar{\psi}\left(\partial_{x}-\partial_{t}\right) \bar{\psi}-m \bar{\psi} \psi\right)
$$


It is a model with only one particle, and with only $\mathbb{Z}_{2}$ internal symmetry, described by a change of sign of the fermion fields. In particular, the fields $\psi$ and $\bar{\psi}$ are both real (hence the corresponding operators in any quantization scheme are Hermitian). The quantization on the line is simple to describe. Fermion operators are given by:

$$
\begin{aligned}
& \psi(x, t)=\frac{1}{2} \sqrt{\frac{m}{\pi}} \int d \theta e^{\theta / 2}\left(a(\theta) e^{i p_{\theta} x-i E_{\theta} t}+a^{\dagger}(\theta) e^{-i p_{\theta} x+i E_{\theta} t}\right), \\
& \bar{\psi}(x, t)=-\frac{i}{2} \sqrt{\frac{m}{\pi}} \int d \theta e^{-\theta / 2}\left(a(\theta) e^{i p_{\theta} x-i E_{\theta} t}-a^{\dagger}(\theta) e^{-i p_{\theta} x+i E_{\theta} t}\right),
\end{aligned}
$$

where the mode operators $a(\theta)$ and their Hermitian conjugate $a^{\dagger}(\theta)$ satisfy the canonical anticommutation relations

$$
\left\{a^{\dagger}(\theta), a\left(\theta^{\prime}\right)\right\}=\delta\left(\theta-\theta^{\prime}\right)
$$

(other anti-commutators vanishing) and where

$$
p_{\theta}=m \sinh \theta, \quad E_{\theta}=m \cosh \theta .
$$

The fermion operators satisfy the equations of motion

$$
\begin{aligned}
\bar{\partial} \psi(x, t) & \equiv \frac{1}{2}\left(\partial_{x}+\partial_{t}\right) \psi=\frac{m}{2} \bar{\psi}, \\
\partial \bar{\psi}(x, t) & \equiv \frac{1}{2}\left(\partial_{x}-\partial_{t}\right) \bar{\psi}=\frac{m}{2} \psi
\end{aligned}
$$

and obey the equal-time anti-commutation relations

$$
\left\{\psi(x, t), \psi\left(x^{\prime}, t\right)\right\}=\delta\left(x-x^{\prime}\right), \quad\left\{\bar{\psi}(x, t), \bar{\psi}\left(x^{\prime}, t\right)\right\}=\delta\left(x-x^{\prime}\right) .
$$

The Hilbert space $\mathcal{H}$ is simply the Fock space over the algebra (7.1) with vacuum vector $\mid$ vac $\rangle$ defined by $a(\theta)|\mathrm{vac}\rangle=0$. Vectors in $\mathcal{H}$ will be denoted by

$$
\left|\theta_{1}, \ldots, \theta_{k}\right\rangle=a^{\dagger}\left(\theta_{1}\right) \cdots a^{\dagger}\left(\theta_{k}\right)|\operatorname{vac}\rangle
$$

A basis is formed by taking, for instance, $\theta_{1}>\cdots>\theta_{k}$. This is exactly the construction described in Section 2, with only one particle and $S(\theta)=-1$. The Hamiltonian is given by

$$
H=\int_{-\infty}^{\infty} d \theta m \cosh \theta a^{\dagger}(\theta) a(\theta)
$$

and has the property of being bounded from below on $\mathcal{H}$ and of generating time translations:

$$
[H, \psi(x, t)]=-i \frac{\partial}{\partial t} \psi(x, t), \quad[H, \bar{\psi}(x, t)]=-i \frac{\partial}{\partial t} \bar{\psi}(x, t) .
$$

In the discussions of the previous sections, we also considered quantization on the circle of circumference $\beta$. It will be convenient to have the description of this quantization for the present model, with anti-periodic (NS) conditions on the fermion fields. The fermion operators evolved in Euclidean time $\tau$ are:

$$
\begin{aligned}
& \hat{\psi}(\mathrm{x}, \tau)=\frac{1}{\sqrt{2 L}} \sum_{n \in \mathbb{Z}+\frac{1}{2}} \frac{e^{\alpha_{n} / 2}}{\sqrt{\cosh \alpha_{n}}}\left(a_{n} e^{i p_{n} \mathrm{x}-E_{n} \tau}+a_{n}^{\dagger} e^{-i p_{n} \mathrm{x}+E_{n} \tau}\right), \\
& \hat{\bar{\psi}}(\mathrm{x}, \tau)=-\frac{i}{\sqrt{2 L}} \sum_{n \in \mathbb{Z}+\frac{1}{2}} \frac{e^{-\alpha_{n} / 2}}{\sqrt{\cosh \alpha_{n}}}\left(a_{n} e^{i p_{n} \mathrm{x}-E_{n} \tau}-a_{n}^{\dagger} e^{-i p_{n} \mathrm{x}+E_{n} \tau}\right),
\end{aligned}
$$


where the discrete mode operators $a_{n}$ and their Hermitian conjugate $a_{n}^{\dagger}$ satisfy the canonical anti-commutation relations

$$
\left\{a_{n}^{\dagger}, a_{n^{\prime}}\right\}=\delta_{n, n^{\prime}}
$$

(other anti-commutators vanishing) and where

$$
\begin{aligned}
& p_{n}=m \sinh \alpha_{n}=\frac{2 \pi n}{L} \quad\left(n \in \mathbb{Z}+\frac{1}{2}\right), \\
& E_{n}=m \cosh \alpha_{n} .
\end{aligned}
$$

The fermion operators satisfy the equations of motion (7.2) as well as the equal-time anticommutation relations (7.3) (with the replacement $\psi \mapsto \hat{\psi}$ and $\bar{\psi} \mapsto \hat{\bar{\psi}}$ ); the latter is simple to derive from the representation

$$
\delta(x)=\frac{1}{L} \sum_{n \in \mathbb{Z}+\frac{1}{2}} e^{i p_{n} x}
$$

of the delta-function, valid on the space of antiperiodic functions on an interval of length $\beta$. The Hilbert space $\mathcal{H}_{\beta}$ is simply the Fock space over the algebra (7.5) with vacuum vector $\left|\operatorname{vac}_{\mathrm{NS}}\right\rangle_{\beta}$ defined by $a_{n}\left|\operatorname{vac}_{\mathrm{NS}}\right\rangle_{\beta}=0$. Vectors in $\mathcal{H}_{\beta}$ will be denoted by

$$
\left|n_{1}, \ldots, n_{k}\right\rangle_{\beta}=a_{n_{1}}^{\dagger} \cdots a_{n_{k}}^{\dagger}\left|\operatorname{vac}_{\mathrm{NS}}\right\rangle_{\beta} .
$$

A basis is formed by taking, for instance, $n_{1}>\cdots>n_{k}$. The Hamiltonian (with vacuum energy) is given by

$$
H_{\beta}=\mathcal{E}_{\mathrm{NS}}+\sum_{n \in \mathbb{Z}+\frac{1}{2}} m \cosh \alpha_{n} a_{n}^{\dagger} a_{n}
$$

and has the property of being bounded from below on $\mathcal{H}_{\beta}$ and of generating time translations:

$$
\left[H_{\beta}, \hat{\psi}(\mathrm{x}, \tau)\right]=\frac{\partial}{\partial \tau} \hat{\psi}(\mathrm{x}, \tau), \quad\left[H_{\beta}, \hat{\bar{\psi}}(\mathrm{x}, \tau)\right]=\frac{\partial}{\partial \tau} \hat{\bar{\psi}}(\mathrm{x}, \tau) .
$$

Our discussion was with the NS sector in mind, but it is not hard to perform the quantization in the $\mathrm{R}$ sector. What will be important for us are relative energies of the NS and $\mathrm{R}$ vacua:

$$
\begin{aligned}
& \mathcal{E}_{\mathrm{NS}} \equiv \mathcal{E}[1 / 2]=\varepsilon-\int_{-\infty}^{\infty} \frac{d \theta}{2 \pi} \cosh \theta \ln \left(1+e^{-m \beta \cosh \theta}\right), \\
& \mathcal{E}_{\mathrm{R}} \equiv \mathcal{E}[0]=\varepsilon-\int_{-\infty}^{\infty} \frac{d \theta}{2 \pi} \cosh \theta \ln \left(1-e^{-m \beta \cosh \theta}\right),
\end{aligned}
$$

where we used the notation of the discussion around (3.9). Here, the vacuum energies of both sectors were calculated in the same regularisation scheme and $\varepsilon$ contain terms that are common to both.

It is worth noting that the normalisation that we took is slightly different from the more standard normalisation in conformal field theory, that makes the fields $\psi$ and $\bar{\psi}$ not real, but with definite phase. With our normalisation, the leading terms of the operator product expansions (OPE's) $\psi(x, t) \psi(0,0)$ and $\bar{\psi}(x, t) \bar{\psi}(0,0)$ are given by

$$
\psi(x, t) \psi(0,0) \sim \frac{i}{2 \pi(x-t)}, \quad \bar{\psi}(x, t) \bar{\psi}(0,0) \sim-\frac{i}{2 \pi(x+t)} .
$$




\subsection{Twist fields}

Two fields are of particular importance: they are two primary twist fields associated to the $\mathbb{Z}_{2}$ symmetry, which we will denote by $\sigma$ and $\mu$ as is customary ${ }^{4}$, the first one being bosonic, the second fermionic. In the sense of quantum chains, the first one is an "order" field, with nonzero vacuum expectation value, the second is a "disorder" field, with zero vacuum expectation value. As we explained in sub-section 3.3, to each of these fields there are two operators on $\mathcal{H}$, which makes four operators: $\sigma_{ \pm}$and $\mu_{ \pm}$. They are fully characterised by the leading terms in the (equal-time) OPE's that are displayed in Appendix A. These leading terms are fixed by the general requirements (3.5) and (3.6), by our choice of branch which says that when fermion operators are placed before the twist-field operators, they are on the same branch no matter the direction of the cut, and by the general "field" product expansion that holds inside correlation functions:

$$
\begin{aligned}
\psi\left(x, t-i 0^{+}\right) \sigma(0, t) & \sim \frac{i}{2 \sqrt{\pi x+i 0^{+}}} \mu(0, t), \quad \psi\left(x, t-i 0^{+}\right) \mu(0, t) \sim \frac{1}{2 \sqrt{\pi x+i 0^{+}}} \sigma(0, t), \\
\bar{\psi}\left(x, t-i 0^{+}\right) \sigma(0, t) & \sim-\frac{i}{2 \sqrt{\pi x-i 0^{+}}} \mu(0, t), \quad \bar{\psi}\left(x, t-i 0^{+}\right) \mu(0, t) \sim \frac{1}{2 \sqrt{\pi x-i 0^{+}}} \sigma(0, t)
\end{aligned}
$$

with branch cuts on $x<0$.

It is worth nothing that the relations of Appendix A are in agreement with the Hermiticity relations $\sigma_{ \pm}^{\dagger}=\sigma_{ \pm}$and $\mu_{ \pm}^{\dagger}= \pm \mu_{ \pm}$.

\subsection{Riemann-Hilbert problem for twisted and untwisted finite-temperature form factors}

\subsubsection{Untwisted case}

In [2], the (untwisted) finite-temperature form factors (4.7) of the twist-field operators above were shown to solve a Riemann-Hilbert problem of the type found at zero temperature, but with important modifications. We repeat here the results.

Consider the function

$$
f_{\eta}\left(\theta_{1}, \ldots, \theta_{k}\right)=F_{+, \ldots,+}^{\mathcal{O}_{\eta}}\left(\theta_{1}, \ldots, \theta_{k} ; \beta\right)
$$

where $\mathcal{O}_{\eta}$ is the operator with branch cut on its right $(\eta=+)$ or on its left $(\eta=-)$ representing any twist field: this can be the order field $\sigma_{ \pm}$or the disorder field $\mu_{ \pm}$, or any of their conformal descendants (that is, fields which reproduce conformal descendants in the massless limit). Conformal descendants include space derivatives, as well as other fields related to action of higher conformal Virasoro modes on twist fields. A way of describing such descendants is by taking the limit $x \rightarrow 0$ of the finite part of the $\mathrm{OPE} \mathcal{O}(x) \sigma_{ \pm}(0)$ or $\mathcal{O}(x) \mu_{ \pm}(0)$, where $\mathcal{O}$ is any bosonic operator formed out of normal-ordered products of fermion operators.

The function $f$ solves the following Riemann-Hilbert problem:

1. Statistics of free particles: $f$ acquires a sign under exchange of any two of the rapidity variables;

2. Quasi-periodicity:

$$
f_{\eta}\left(\theta_{1}, \ldots, \theta_{j}+2 i \pi, \ldots, \theta_{k}\right)=-f\left(\theta_{1}, \ldots, \theta_{j}, \ldots, \theta_{k}\right), \quad j=1, \ldots, k
$$

\footnotetext{
${ }^{4}$ In the present section, the symbol $\sigma$ does not denote a generic twist field, but rather the primary twist field as described here.
} 
3. Analytic structure: $f$ is analytic as function of all of its variables $\theta_{j}, j=1, \ldots, k$ everywhere on the complex plane except at simple poles. In the region $\operatorname{Im}\left(\theta_{j}\right) \in[-i \pi, i \pi]$, $j=1, \ldots, k$, its analytic structure is specified as follows:

(a) Thermal poles and zeroes: $f_{\eta}\left(\theta_{1}, \ldots, \theta_{k}\right)$ has poles at

$$
\theta_{j}=\alpha_{n}-\eta \frac{i \pi}{2}, \quad n \in \mathbb{Z}, \quad j=1, \ldots, k
$$

and has zeroes at

$$
\theta_{j}=\alpha_{n}-\eta \frac{i \pi}{2}, \quad n \in \mathbb{Z}+\frac{1}{2}, \quad j=1, \ldots, k,
$$

where $\alpha_{n}$ are defined in (7.6) (and, of course, we use this definition for any $n$ );

(b) Kinematical poles: $f_{\eta}\left(\theta_{1}, \ldots, \theta_{k}\right)$ has poles, as a function of $\theta_{k}$, at $\theta_{j} \pm i \pi, j=$ $1, \ldots, k-1$ with residues given by

$$
f_{\eta}\left(\theta_{1}, \ldots, \theta_{k}\right) \sim \pm \eta \frac{(-1)^{k-j}}{\pi} \frac{1+e^{-\beta E_{\theta_{j}}}}{1-e^{-\beta E_{\theta_{j}}}} \frac{f_{\eta}\left(\theta_{1}, \ldots, \hat{\theta}_{j}, \ldots, \theta_{k-1}\right)}{\theta_{k}-\theta_{j} \mp i \pi} .
$$

In order to have other finite-temperature form factors than those with all positive charges, one more relation needs to be used. We have:

4. Crossing symmetry:

$$
F_{\epsilon_{1}, \ldots, \epsilon_{j}, \ldots, \epsilon_{k}}^{\mathcal{O}_{\eta}}\left(\theta_{1}, \ldots, \theta_{j}+i \pi, \ldots, \theta_{k} ; \beta\right)=i F_{\epsilon_{1}, \ldots,-\epsilon_{j}, \ldots, \epsilon_{k}}^{\mathcal{O}_{\eta}}\left(\theta_{1}, \ldots, \theta_{j}, \ldots, \theta_{k} ; \beta\right)
$$

The name "crossing symmetry" is inspired by the zero-temperature case (and it is not to be confused with the simpler "crossing relations" introduced in (4.4), (4.5), (6.2), (6.3)). To make it more obvious, define the functions

$$
f_{\eta}\left(\theta_{1}^{\prime}, \ldots, \theta_{l}^{\prime} \mid \theta_{1}, \ldots, \theta_{k}\right)=\left(d_{+, \ldots,+}\left(\theta_{1}^{\prime}, \ldots, \theta_{l}^{\prime}\right), \phi_{L}\left(\mathcal{O}_{\eta}(0,0)\right) d_{+, \ldots,+}\left(\theta_{1}, \ldots, \theta_{k}\right) .\right.
$$

These are in fact distributions, and can be decomposed in terms supported at separated rapidities $\theta_{i}^{\prime} \neq \theta_{j}, \forall i, j$, and terms supported at colliding rapidities, $\theta_{i}^{\prime}=\theta_{j}$ for some $i$ and $j$. We will denote the former by $f_{\eta}^{\text {sep. }}\left(\theta_{1}^{\prime}, \ldots, \theta_{l}^{\prime} \mid \theta_{1}, \ldots, \theta_{k}\right)$, and the latter by $f_{\eta}^{\text {coll. }}\left(\theta_{1}^{\prime}, \ldots, \theta_{l}^{\prime} \mid \theta_{1}, \ldots, \theta_{k}\right)$. Under integration over rapidity variables, the former gives principal value integrals. Recalling the property (6.2), we have

$$
f_{\eta}^{\text {sep. }}\left(\theta_{1}^{\prime}, \ldots, \theta_{l}^{\prime} \mid \theta_{1}, \ldots, \theta_{k}\right)=F_{+, \ldots,+,-, \ldots,-}^{\mathcal{O}_{\eta}}\left(\theta_{1}, \ldots, \theta_{k}, \theta_{l}^{\prime}, \ldots, \theta_{1}^{\prime} ; \beta\right)
$$

for $\left(\theta_{i}^{\prime} \neq \theta_{j} \forall i \in\{1, \ldots, l\}, j \in\{1, \ldots, k\}\right)$, where on the right-hand side, there are $k$ positive charges $(+)$, and $l$ negative charges $(-)$. Analytically extending from its support the distribution $f_{\eta}^{\text {sep. }}$ to a function of complex rapidity variables, crossing symmetry can then be written

$$
\begin{aligned}
& f_{\eta}^{\text {sep. }}\left(\theta_{1}^{\prime}, \ldots, \theta_{l}^{\prime} \mid \theta_{1}, \ldots, \theta_{k}+i \pi\right)=i f_{\eta}^{\text {sep. }}\left(\theta_{1}^{\prime}, \ldots, \theta_{l}^{\prime}, \theta_{k} \mid \theta_{1}, \ldots, \theta_{k-1}\right), \\
& f_{\eta}^{\text {sep. }}\left(\theta_{1}^{\prime}, \ldots, \theta_{l}^{\prime}+i \pi \mid \theta_{1}, \ldots, \theta_{k}\right)=i f_{\eta}^{\text {sep. }}\left(\theta_{1}^{\prime}, \ldots, \theta_{l-1}^{\prime} \mid \theta_{1}, \ldots, \theta_{k}, \theta_{l}^{\prime}\right),
\end{aligned}
$$

which justifies its name.

It is worth mentioning that the distributive terms corresponding to colliding rapidities satisfy a set of recursive equations: 
5. Colliding part of matrix elements:

$$
\begin{aligned}
& f_{\eta}^{\text {coll. }}\left(\theta_{1}^{\prime}, \ldots, \theta_{l}^{\prime} \mid \theta_{1}, \ldots, \theta_{k}\right) \\
& \quad=\sum_{i=1}^{l} \sum_{j=1}^{k}(-1)^{l+k-i-j} \frac{1+e^{-\beta E_{\theta_{j}}}}{1-e^{\beta E_{\theta_{j}}}} \delta\left(\theta_{i}^{\prime}-\theta_{j}\right) f_{\eta}\left(\theta_{1}^{\prime}, \ldots, \hat{\theta}_{i}^{\prime}, \ldots, \theta_{l}^{\prime} \mid \theta_{1}, \ldots, \hat{\theta}_{j}, \ldots, \theta_{k}\right) .
\end{aligned}
$$

Note that the colliding part vanishes in the limit of zero temperature, $\beta \rightarrow \infty$. Finally, it is instructive to re-write the distribution $f_{\eta}\left(\theta_{1}^{\prime}, \ldots, \theta_{l}^{\prime} \mid \theta_{1}, \ldots, \theta_{k}\right)$ as an analytical function with slightly shifted rapidities, plus a distribution, using the relations

$$
\frac{1}{\theta \mp i 0^{+}}= \pm i \pi \delta(\theta)+\underline{\mathrm{P}}\left(\frac{1}{\theta}\right)
$$

where $\underline{P}$ means that we must take the principal value integral under integration. Defining the disconnected part $f_{\eta}^{\text {disconn. }}\left(\theta_{1}^{\prime}, \ldots, \theta_{l}^{\prime} \mid \theta_{1}, \ldots, \theta_{k}\right)$ of the matrix element (7.9) as

$$
\begin{aligned}
f_{\eta}\left(\theta_{1}^{\prime}, \ldots, \theta_{l}^{\prime} \mid \theta_{1}, \ldots, \theta_{k}\right)= & f_{\eta}^{\text {sep. }}\left(\theta_{1}^{\prime}-\eta i 0^{+}, \ldots, \theta_{l}^{\prime}-\eta i 0^{+} \mid \theta_{1}, \ldots, \theta_{k}\right) \\
& +f_{\eta}^{\text {disconn. }}\left(\theta_{1}^{\prime}, \ldots, \theta_{l}^{\prime} \mid \theta_{1}, \ldots, \theta_{k}\right)
\end{aligned}
$$

where again we analytically extend from its support the distribution $f_{\eta}^{\text {sep. }}$ to a function of complex rapidity variables, we find that the disconnected part satisfies the recursion relations

$$
\begin{aligned}
& f_{\eta}^{\text {disconn. }}\left(\theta_{1}^{\prime}, \ldots, \theta_{l}^{\prime} \mid \theta_{1}, \ldots, \theta_{k}\right) \\
& \quad=\sum_{i=1}^{l} \sum_{j=1}^{k}(-1)^{l+k-i-j}\left(1+e^{-\beta E_{\theta_{j}}}\right) \delta\left(\theta_{i}^{\prime}-\theta_{j}\right) f_{\eta}\left(\theta_{1}^{\prime}, \ldots, \hat{\theta}_{i}^{\prime}, \ldots, \theta_{l}^{\prime} \mid \theta_{1}, \ldots, \hat{\theta}_{j}, \ldots, \theta_{k}\right) .
\end{aligned}
$$

Note that the factor $\left(1+e^{-\beta E_{\theta_{j}}}\right) \delta\left(\theta_{i}^{\prime}-\theta_{j}\right)$ appearing inside the double sum is just the overlap $\left(d_{+}\left(\theta_{i}^{\prime}\right), d_{+}\left(\theta_{j}\right)\right)$, so that the equation above can be naturally represented as a "sum of disconnected diagrams." This equation is, in fact, consequence of the general relation (6.6).

\subsubsection{Twisted case}

The twisted case was not considered in [2], but can be obtained from the same arguments.

There is no $U(1)$ invariance, but we can still twist by the $\mathbb{Z}_{2}$ symmetry. Hence, we consider twisted finite-temperature form factors (5.6) with $\omega=\frac{1}{2}$. The derivation of [2] for the RiemannHilbert problem can easily be adapted to this case, and the results are as follows.

Consider the function

$$
f_{\eta}\left(\theta_{1}, \ldots, \theta_{k}\right)={ }^{\frac{1}{2}} F_{+, \ldots,+}^{\mathcal{O}_{\eta}}\left(\theta_{1}, \ldots, \theta_{k} ; \beta\right),
$$

where $\mathcal{O}_{\eta}$ is the operator with branch cut on its right $(\eta=+)$ or on its left $(\eta=-)$ representing a twist field. The function $f$ solves the following Riemann-Hilbert problem:

1. Statistics of free particles: $f$ acquires a sign under exchange of any two of the rapidity variables;

2. Quasi-periodicity:

$$
f_{\eta}\left(\theta_{1}, \ldots, \theta_{j}+2 i \pi, \ldots, \theta_{k}\right)=-f_{\eta}\left(\theta_{1}, \ldots, \theta_{j}, \ldots, \theta_{k}\right), \quad j=1, \ldots, k
$$

3. Analytic structure: $f$ is analytic as function of all of its variables $\theta_{j}, j=1, \ldots, k$ everywhere on the complex plane except at simple poles. In the region $\operatorname{Im}\left(\theta_{j}\right) \in[-i \pi, i \pi]$, $j=1, \ldots, k$, its analytic structure is specified as follows: 
(a) Thermal poles and zeroes: $f_{\eta}\left(\theta_{1}, \ldots, \theta_{k}\right)$ has poles at

$$
\theta_{j}=\alpha_{n}-\eta \frac{i \pi}{2}, \quad n \in \mathbb{Z}+\frac{1}{2}, \quad j=1, \ldots, k
$$

and has zeroes at

$$
\theta_{j}=\alpha_{n}-\eta \frac{i \pi}{2}, \quad n \in \mathbb{Z}, \quad j=1, \ldots, k ;
$$

(b) Kinematical poles: $f_{\eta}\left(\theta_{1}, \ldots, \theta_{k}\right)$ has poles, as a function of $\theta_{k}$, at $\theta_{j} \pm i \pi, j=$ $1, \ldots, k-1$ with residues given by

$$
f_{\eta}\left(\theta_{1}, \ldots, \theta_{k} ; L\right) \sim \pm \eta \frac{(-1)^{k-j}}{\pi} \frac{1-e^{-\beta E_{\theta_{j}}}}{1+e^{-\beta E_{\theta_{j}}}} \frac{f_{\eta}\left(\theta_{1}, \ldots, \hat{\theta}_{j}, \ldots, \theta_{k-1}\right)}{\theta_{k}-\theta_{j} \mp i \pi} .
$$

Again, in order to have other finite-temperature form factors than those with all positive charges, one more relation needs to be used. We have:

4. Crossing symmetry:

$$
{ }^{\frac{1}{2}} F_{\epsilon_{1}, \ldots, \epsilon_{j}, \ldots, \epsilon_{k}}^{\mathcal{O}_{\eta}}\left(\theta_{1}, \ldots, \theta_{j}+i \pi, \ldots, \theta_{k} ; \beta\right)=i^{\frac{1}{2}} F_{\epsilon_{1}, \ldots,-\epsilon_{j}, \ldots, \epsilon_{k}}^{\mathcal{O}_{\eta}}\left(\theta_{1}, \ldots, \theta_{j}, \ldots, \theta_{k} ; \beta\right) .
$$

Moreover, matrix elements

$$
f_{\eta}\left(\theta_{1}^{\prime}, \ldots, \theta_{l}^{\prime} \mid \theta_{1}, \ldots, \theta_{k}\right)=\left(d_{+, \ldots,+}\left(\theta_{1}^{\prime}, \ldots, \theta_{l}^{\prime}\right), \phi_{L}\left(\mathcal{O}_{\eta}(0,0)\right) d_{+, \ldots,+}\left(\theta_{1}, \ldots, \theta_{k}\right)_{\frac{1}{2}}\right.
$$

can again be decomposed in terms supported at separated rapidities $\theta_{i}^{\prime} \neq \theta_{j}, \forall i, j$ (which give principal value integrals under integration), and terms supported at colliding rapidities, $\theta_{i}^{\prime}=\theta_{j}$ for some $i$ and $j$, denoted respectively by $f_{\eta}^{\text {sep. }}\left(\theta_{1}^{\prime}, \ldots, \theta_{l}^{\prime} \mid \theta_{1}, \ldots, \theta_{k}\right)$ and $f_{\eta}^{\text {coll. }}\left(\theta_{1}^{\prime}, \ldots, \theta_{l}^{\prime} \mid\right.$ $\left.\theta_{1}, \ldots, \theta_{k}\right)$. Recalling the property $(6.2)$, we have

$$
f_{\eta}^{\text {sep. }}\left(\theta_{1}^{\prime}, \ldots, \theta_{l}^{\prime} \mid \theta_{1}, \ldots, \theta_{k}\right)=\frac{1}{2} F_{+, \ldots,+,-, \ldots,-}^{\mathcal{O}_{\eta}}\left(\theta_{1}, \ldots, \theta_{k}, \theta_{l}^{\prime}, \ldots, \theta_{1}^{\prime} ; \beta\right)
$$

for $\left(\theta_{i}^{\prime} \neq \theta_{j} \forall i \in\{1, \ldots, l\}, j \in\{1, \ldots, k\}\right)$, where on the right-hand side, there are $k$ positive charges $(+)$, and $l$ negative charges $(-)$. The distributive terms corresponding to colliding rapidities satisfy again a set of recursive equations, now modified by the twisting:

5. Colliding part of matrix elements:

$$
\begin{aligned}
f_{\eta}^{\text {coll. }} & \left(\theta_{1}^{\prime}, \ldots, \theta_{l}^{\prime} \mid \theta_{1}, \ldots, \theta_{k}\right) \\
& =\sum_{i=1}^{l} \sum_{j=1}^{k}(-1)^{l+k-i-j} \frac{1-e^{-\beta E_{\theta_{j}}}}{1+e^{\beta E_{\theta_{j}}}} \delta\left(\theta_{i}^{\prime}-\theta_{j}\right) f_{\eta}\left(\theta_{1}^{\prime}, \ldots, \hat{\theta}_{i}^{\prime}, \ldots, \theta_{l}^{\prime} \mid \theta_{1}, \ldots, \hat{\theta}_{j}, \ldots, \theta_{k}\right) .
\end{aligned}
$$

Finally, we can again re-write the distribution $f_{\eta}\left(\theta_{1}^{\prime}, \ldots, \theta_{l}^{\prime} \mid \theta_{1}, \ldots, \theta_{k}\right)$ as an analytical function with slightly shifted rapidities, plus a distribution, using the relations (7.10). Defining the disconnected part $f_{\eta}^{\text {disconn. }}\left(\theta_{1}^{\prime}, \ldots, \theta_{l}^{\prime} \mid \theta_{1}, \ldots, \theta_{k}\right)$ of the matrix element $(7.9)$ as

$$
\begin{aligned}
f_{\eta}\left(\theta_{1}^{\prime}, \ldots, \theta_{l}^{\prime} \mid \theta_{1}, \ldots, \theta_{k}\right)= & f_{\eta}^{\text {sep. }}\left(\theta_{1}^{\prime}-\eta i 0^{+}, \ldots, \theta_{l}^{\prime}-\eta i 0^{+} \mid \theta_{1}, \ldots, \theta_{k}\right) \\
& +f_{\eta}^{\text {disconn. }}\left(\theta_{1}^{\prime}, \ldots, \theta_{l}^{\prime} \mid \theta_{1}, \ldots, \theta_{k}\right),
\end{aligned}
$$

where again we analytically extend from its support the distribution $f_{\eta}^{\text {sep. }}$ to a function of complex rapidity variables, we find that the disconnected part satisfies the recursion relations

$$
\begin{aligned}
& f_{\eta}^{\text {disconn. }}\left(\theta_{1}^{\prime}, \ldots, \theta_{l}^{\prime} \mid \theta_{1}, \ldots, \theta_{k}\right) \\
& \quad=\sum_{i=1}^{l} \sum_{j=1}^{k}(-1)^{l+k-i-j}\left(1-e^{-\beta E_{\theta_{j}}}\right) \delta\left(\theta_{i}^{\prime}-\theta_{j}\right) f_{\eta}\left(\theta_{1}^{\prime}, \ldots, \hat{\theta}_{i}^{\prime}, \ldots, \theta_{l}^{\prime} \mid \theta_{1}, \ldots, \hat{\theta}_{j}, \ldots, \theta_{k}\right) .
\end{aligned}
$$




\subsubsection{Other local fields}

It is worth noting that points 1,2 and 4 are in fact also valid for fields that are local with respect to $\psi$ and $\bar{\psi}$. The analytic structure, point 3, for such fields, is much simpler: the finitetemperature form factors are entire functions of all rapidities. In fact, the finite-temperature form factors of $\psi$ and $\bar{\psi}$ themselves are exactly equal to their zero-temperature form factors, and for other fields, a phenomenon of mixing occurs, as described in [2] and as can be calculated using the techniques of Section 6 .

\subsubsection{Differences with zero-temperature Riemann-Hilbert problems, and some explanations}

There are three main differences between the Riemann-Hilbert problems stated in this subsection, and the Riemann-Hilbert problems solved by zero-temperature form factors, reviewed in Section 2. First, there are, in the former, so-called "thermal" poles and zeroes. They are in fact consequences of the semi-locality of the operators with respect to the fundamental fermion fields, and play the role of "changing the sector" of the excited states when integrals are deformed to reproduce the form factor expansion in the quantization on the circle. Indeed, they displace the poles of the measure in order to reproduce the right set of discrete momenta.

Second, the kinematical residue has an additional factor. This factor, in fact, is closely related to the presence of the thermal poles and zeroes.

Finally, there is a subtle but important difference: the quasi-periodicity equation has a sign difference. Essentially, the quasi-periodicity equation that we have at finite-temperature is exactly the one we would have at zero-temperature with fields that are local with respect to the fermion field. This difference is again due to the presence of the thermal poles and zeroes. More precisely, in the limit of zero temperature, the finite-temperature form factors converge to the zero temperature one only in the strip $\operatorname{Im}(\theta) \in]-\pi / 2, \pi / 2[$. At the $\operatorname{lines} \operatorname{Im}(\theta)= \pm \pi / 2$ (the sign depending on which excitation type and form factor we are looking at), there is an accumulation of poles and zeroes that produces a cut. The quasi-periodicity equation of zero temperature comes from the analytical continuation through this cut. Note that it is this analytical continuation that recovers rotation invariance in Euclidean plane, an invariance which is broken by the cylindrical geometry at finite temperature.

We would like to mention, in relation to the breaking of Euclidean rotation invariance, that yet crossing symmetry, point 4 , is valid. It is in fact a consequence of the fact that the deformation of the contours, as explained in Subsection 4.4, should give residues at the poles of the measure occurring in (4.6). These residues come from two contributions: the contribution of the displaced $\theta$ contour associated to $\epsilon=+$, and that associated to $\epsilon=-$. That these two contributions should give a residue impose certain conditions on the value of the finite-temperature form factors: they should correspond to contours in opposite direction and on opposite sides of the same pole. From this and from knowing that all finite-temperature form factors of the fermion fields satisfy crossing symmetry, one concludes that crossing symmetry holds for all local fields.

\subsection{Results for twisted and untwisted finite-temperature form factors}

Again, we repeat here the results of [2], and generalise them to the twisted case. Note that the method of computing one-particle finite-temperature form factors by solving the RiemannHilbert problem with this asymptotic is very similar to the method used by Fonseca and Zamolodchikov [21] for calculating form factors on the circle.

For the order and disorder operators, $\sigma_{ \pm}$and $\mu_{ \pm}$the solutions to the Riemann-Hilbert problems above are completely fixed (up to a normalization) by the asymptotic $\sim O(1)$ at $\theta \rightarrow \pm \infty$, imposed by the fact that they are primary fields of spin 0 . 
For the one-particle finite-temperature form factor of the disorder operator with a branch cut on its right, the solution is

$$
F_{ \pm}^{\mu_{+}}(\theta ; \beta)=e^{ \pm \frac{i \pi}{4}} C(\beta) \exp \left[\mp \int_{-\infty \mp i 0^{+}}^{\infty \mp i 0^{+}} \frac{d \theta^{\prime}}{2 \pi i} \frac{1}{\sinh \left(\theta-\theta^{\prime}\right)} \ln \left(\frac{1+e^{-\beta E_{\theta^{\prime}}}}{1-e^{-\beta E_{\theta^{\prime}}}}\right)\right]
$$

for some real constant $C(\beta)$. This is in agreement with the Hermiticity of $\mu_{+}$, which gives $\left(F_{ \pm}^{\mu_{+}}(\theta ; \beta)\right)^{*}=F_{\mp}^{\mu_{+}}(\theta ; \beta)$ for $\theta$ real. Using

$$
\frac{1}{\sinh \left(\theta-\left(\theta^{\prime} \pm i 0^{+}\right)\right)}= \pm i \pi \delta\left(\theta-\theta^{\prime}\right)+\underline{\mathrm{P}}\left(\frac{1}{\sinh \left(\theta-\theta^{\prime}\right)}\right)
$$

this can also be written

$$
F_{ \pm}^{\mu_{+}}(\theta ; \beta)=C(\beta) e^{ \pm \frac{i \pi}{4}} \sqrt{\frac{1+e^{-\beta E_{\theta}}}{1-e^{-\beta E_{\theta}}}} \exp \left[\mp \int_{-\infty}^{\infty} \frac{d \theta^{\prime}}{2 \pi i} \underline{\mathrm{P}}\left(\frac{1}{\sinh \left(\theta-\theta^{\prime}\right)}\right) \ln \left(\frac{1+e^{-\beta E_{\theta^{\prime}}}}{1-e^{-\beta E_{\theta^{\prime}}}}\right)\right] .
$$

That this is a solution can be checked by verifying the asymptotic $F_{ \pm}^{\mu_{+}}(\theta ; \beta) \sim e^{ \pm \frac{i \pi}{4}} C(\beta)$ as $|\theta| \rightarrow \infty$, and by verifying that the functions $F_{ \pm}^{\mu_{+}}(\theta ; \beta)$ have poles and zeros at the proper positions. Positions of poles and zeros are the values of $\theta$ such that when analytically continued from real values, a pole at $\sinh \left(\theta-\theta^{\prime}\right)=0$ in the integrand of (7.11) and one of the logarithmic branch points pinch the $\theta^{\prime}$ contour of integration. The fact that these positions correspond to poles and zeros can be deduced most easily from the functional relation

$$
F_{ \pm}^{\mu_{+}}(\theta ; \beta) F_{ \pm}^{\mu_{+}}(\theta \pm i \pi ; \beta)= \pm i C(\beta)^{2} \frac{1+e^{-\beta E_{\theta}}}{1-e^{-\beta E_{\theta}}}
$$

Note that this implies the quasi-periodicity property

$$
F_{ \pm}^{\mu_{+}}(\theta+2 i \pi ; \beta)=-F_{ \pm}^{\mu_{+}}(\theta ; \beta) .
$$

It is also easy to see that the crossing symmetry relation is satisfied.

For the operator $\mu_{-}$with a branch cut on its left, one can check similarly that the function

$$
F_{ \pm}^{\mu_{-}}(\theta ; \beta)=F_{ \pm}^{\mu_{+}}(\theta-i \pi ; \beta)=-i F_{\mp}^{\mu_{+}}(\theta ; \beta)
$$

solves the Riemann-Hilbert problem of Paragraph 7.3.1 with $\eta=-$. Explicitly,

$$
F_{ \pm}^{\mu_{-}}(\theta ; \beta)=-i e^{\mp \frac{i \pi}{4}} C(\beta) \exp \left[ \pm \int_{-\infty \pm i 0^{+}}^{\infty \pm i 0^{+}} \frac{d \theta^{\prime}}{2 \pi i} \frac{1}{\sinh \left(\theta-\theta^{\prime}\right)} \ln \left(\frac{1+e^{-\beta E_{\theta^{\prime}}}}{1-e^{-\beta E_{\theta^{\prime}}}}\right)\right] .
$$

In particular, we observe that $\left(F_{ \pm}^{\mu_{-}}(\theta ; \beta)\right)^{*}=-F_{\mp}^{\mu_{-}}(\theta ; \beta)$, which is in agreement with the antiHermiticity of the operator $\mu_{-}$. Note that we chose the same constant $C(\beta)$ as a normalization for both $F_{ \pm}^{\mu_{-}}$and $F_{ \pm}^{\mu_{+}}$. This is not a consequence of the Riemann-Hilbert problem, but can be checked by explicitly calculating the normalisation. The normalisation was calculated in [2], and is given by

$$
C(\beta)=\frac{\langle\langle\sigma\rangle\rangle_{\beta}}{\sqrt{2 \pi}}
$$

where the average $\langle\langle\sigma\rangle\rangle_{\beta}$ was calculated in [28] (the average at zero-temperature (that is, $\beta \rightarrow \infty$ ) can be found in [45]) and is given by

$$
m^{\frac{1}{8}} 2^{\frac{1}{12}} e^{-\frac{1}{8}} A^{\frac{3}{2}} \exp \left[\frac{(m \beta)^{2}}{2} \iint_{-\infty}^{\infty} \frac{d \theta_{1} d \theta_{2}}{(2 \pi)^{2}} \frac{\sinh \theta_{1} \sinh \theta_{2}}{\sinh \left(m \beta \cosh \theta_{1}\right) \sinh \left(m \beta \cosh \theta_{2}\right)}\right.
$$




$$
\left.\times \ln \left|\left(\operatorname{coth} \frac{\theta_{1}-\theta_{2}}{2}\right)\right|\right]
$$

where $A$ is Glaisher's constant. Essentially, this normalisation is evaluated by computing the leading of $\left\langle\left\langle\psi(x, 0) \mu_{-}(0,0)\right\rangle\right\rangle_{\beta}$ as $x \rightarrow 0^{+}$, and the leading of $\left\langle\left\langle\mu_{+}(0,0) \psi(x, 0)\right\rangle\right\rangle_{\beta}$ as $x \rightarrow 0^{-}$, using the form factor expansions; in both cases, it is important to approach the point $x=0$ from a region that is away from the cut.

Multi-particle finite-temperature form factors can be easily constructed from the well-known zero-temperature form factors (first calculated in [9]), by adjoining "leg factors", which are just normalized one-particle finite-temperature form factors:

$$
F_{+, \ldots,+}^{\mathcal{O}_{+}}\left(\theta_{1}, \ldots, \theta_{k} ; \beta\right)=i^{\left[\frac{k}{2}\right]}\langle\langle\sigma\rangle\rangle_{\beta}\left(\prod_{j=1}^{k} \frac{F_{+}^{\mu_{+}}\left(\theta_{j} ; \beta\right)}{\langle\langle\sigma\rangle\rangle_{\beta}}\right) \prod_{1 \leq i<j \leq k} \tanh \left(\frac{\theta_{j}-\theta_{i}}{2}\right)
$$

where $\mathcal{O}_{+}$is $\sigma_{+}$if $k$ is even, and $\mu_{+}$if $k$ is odd. The symbol [k/2] equals the greatest integer smaller than or equal to $k / 2$. This satisfies the condition on thermal poles and zeroes simply from the properties of the leg factors, and it can be verified that this satisfies the quasi-periodicity condition and the kinematical pole condition, Point 2 and Point 3b of Subsection 7.3.1, respectively. Using crossing symmetry, Point 4, it is a simple matter to obtain the formula for other values of the charges:

$$
F_{\epsilon_{1}, \ldots, \epsilon_{k}}^{\mathcal{O}_{+}}\left(\theta_{1}, \ldots, \theta_{k} ; \beta\right)=i^{\left[\frac{k}{2}\right]}\langle\langle\sigma\rangle\rangle_{\beta}\left(\prod_{j=1}^{k} \frac{F_{\epsilon_{j}}^{\mu_{+}}\left(\theta_{j} ; \beta\right)}{\langle\langle\sigma\rangle\rangle_{\beta}}\right) \prod_{1 \leq i<j \leq k}\left(\tanh \left(\frac{\theta_{j}-\theta_{i}}{2}\right)\right)^{\epsilon_{i} \epsilon_{j}}
$$

Similarly, we have

$$
F_{\epsilon_{1}, \ldots, \epsilon_{k}}^{\mathcal{O}_{-}}\left(\theta_{1}, \ldots, \theta_{k} ; \beta\right)=i^{\left[\frac{k}{2}\right]}\left\langle\langle\sigma \sigma\rangle_{\beta}\left(\prod_{j=1}^{k} \frac{F_{\epsilon_{j}}^{\mu_{-}}\left(\theta_{j} ; \beta\right)}{\langle\langle\sigma\rangle\rangle_{\beta}}\right) \prod_{1 \leq i<j \leq k}\left(\tanh \left(\frac{\theta_{j}-\theta_{i}}{2}\right)\right)^{\epsilon_{i} \epsilon_{j}},\right.
$$

where $\mathcal{O}_{-}$is $\sigma_{-}$if $k$ is even, and $\mu_{-}$if $k$ is odd.

Finally, twisted one-particle finite-temperature form factors can easily be obtained by solving the Riemann-Hilbert problem of Paragraph 7.3.2 as follows:

$$
\frac{1}{2} F_{\epsilon}^{\mu_{\eta}}(\theta)=\frac{\epsilon i C(\beta)^{2}}{F_{\epsilon}^{\mu_{\eta}}(\theta)}
$$

These functions have the correct analytic structure, they satisfy the crossing symmetry relation (point 4), and their normalisation is the correct one that can be deduced from the fact that the leading of $\left(\psi(x, 0), \mu_{-}(0,0)\right)_{\frac{1}{2}}$ as $x \rightarrow 0^{+}$, and the leading of $\left(\mu_{+}(0,0), \psi(x, 0)\right)_{\frac{1}{2}}$ as $x \rightarrow 0^{-}$, are the same as in the untwisted case. Twisted multi-particle form factors can also be obtained in a simple way:

$$
\begin{aligned}
& \quad \frac{1}{2} F_{\epsilon_{1}, \ldots, \epsilon_{k}}^{\mathcal{O}_{\eta}}\left(\theta_{1}, \ldots, \theta_{k} ; \beta\right) \\
& \quad=i^{\left[\frac{k}{2}\right]}\left\langle\langle\langle\sigma\rangle\rangle_{\beta}\left(\prod_{j=1}^{k} \frac{\frac{1}{2} F_{\epsilon_{j}}^{\mu_{\eta}}\left(\theta_{j} ; \beta\right)}{\langle\langle\sigma\rangle\rangle_{\beta}}\right) \prod_{1 \leq i<j \leq k}\left(\tanh \left(\frac{\theta_{j}-\theta_{i}}{2}\right)\right)^{\epsilon_{i} \epsilon_{j}},\right.
\end{aligned}
$$

where $\mathcal{O}_{ \pm}$is $\sigma_{ \pm}$if $k$ is even, and $\mu_{ \pm}$if $k$ is odd. 


\subsection{Form factors on the circle from finite-temperature form factors}

As explained in Subsection 4.4, there is a relation between finite-temperature form factors and form factors in the quantization on the circle. In the present case of the Majorana theory, this relation was written explicitly in [2], and was proven by independent means. A slight extension to the twisted case gives it as follows:

$$
\begin{aligned}
\beta\left\langle\tilde{n}_{1}, \ldots,\right. & \left., \tilde{n}_{l}|\hat{\mathcal{O}}(0,0)| n_{1}, \ldots, n_{k}\right\rangle_{\beta} \\
= & e^{-\frac{i \pi s}{2}}\left(\frac{2 \pi}{m L}\right)^{\frac{k+l}{2}}\left(\prod_{j=1}^{l} \frac{1}{\sqrt{\cosh \left(\alpha_{\tilde{n}_{j}}\right)}}\right)\left(\prod_{j=1}^{k} \frac{1}{\sqrt{\cosh \left(\alpha_{n_{j}}\right)}}\right) \\
& \times{ }^{\omega} F_{+, \ldots,+,-, \ldots,-}^{\mathcal{O}}\left(\alpha_{n_{1}}+\frac{i \pi}{2}, \ldots, \alpha_{n_{k}}+\frac{i \pi}{2}, \alpha_{\tilde{n}_{l}}+\frac{i \pi}{2}, \ldots, \alpha_{\tilde{n}_{1}}+\frac{i \pi}{2} ; \beta\right),
\end{aligned}
$$

where there are $k$ positive charges and $l$ negative charges in the indices of ${ }^{\omega} F^{\mathcal{O}}$, and where $\alpha_{n}$ are defined in (7.6). Here, $s$ is the spin of $\mathcal{O}$. This formula is valid for any excited states in the sector above $\left|\operatorname{vac}_{\frac{1}{2}+\omega}\right\rangle$ (see the discussion around (3.9)). That is, if $\omega=0$, it is valid for excited states in the NS vacuum, hence with $n_{i}, \tilde{n}_{i} \in \mathbb{Z}+\frac{1}{2}$. For $\omega=\frac{1}{2}$, it is valid for excited states in the $\mathrm{R}$ vacuum, hence with $n_{i}, \tilde{n}_{i} \in \mathbb{Z}$.

When $\mathcal{O}$ is a twist field, its associated branch cut changes the sector of the bra or the ket, hence formula (7.18) can then be applied only if one of the bra or the ket is the vacuum, and if the branch cut associated to the twist field is chosen so that this vacuum is in the opposite sector (in order to keep the excited states in the same sector). If $\omega=0$, the vacuum will then be in the $R$ sector, and if $\omega=\frac{1}{2}$, it will be in the $N S$ sector. For a branch cut to the right, it is the bra that must be chosen as this vacuum, whereas for a branch cut to the left, it is the ket.

It is easy to check, using (7.18), that the formulas above for finite-temperature form factors reproduce the known form factors on the circle [19, 20, 21].

\subsection{Two-point functions, Fredholm determinants and scaling limit of the quantum Ising model}

The finite-temperature form factor expansion (5.5) now gives explicit expansions for finitetemperature two-point functions of twist fields at $x>0$ :

$$
\begin{aligned}
& \left\langle\left\langle\sigma_{+}(x, t) \sigma_{-}(0,0)\right\rangle\right\rangle_{\beta}^{\omega}=e^{\left(\mathcal{E}[\omega]-\mathcal{E}\left[\frac{1}{2}+\omega\right]\right) x} \\
& \times \sum_{\substack{k=0 \\
k \text { even }}}^{\infty} \sum_{\epsilon_{1}, \ldots, \epsilon_{k}= \pm} \int_{\left\{\operatorname{Im}\left(\theta_{j}\right)=\epsilon_{j} 0^{+}\right\}} \frac{d \theta_{1} \cdots d \theta_{k} e^{\sum_{j=1}^{k} \epsilon_{j}\left(i m x \sinh \theta_{j}-i m t \cosh \theta_{j}\right)}}{k ! \prod_{j=1}^{k}\left(1+e^{2 \pi i \omega} e^{-\epsilon_{j} m \beta \cosh \theta_{j}}\right)} \\
& \times i^{k} \prod_{j=1}^{k}\left({ }^{\omega} F_{\epsilon_{j}}^{\mu_{+}}\left(\theta_{j} ; L\right)\right)^{2} \prod_{1 \leq i<j \leq k} \tanh \left(\frac{\theta_{j}-\theta_{i}}{2}\right)^{2 \epsilon_{i} \epsilon_{j}}
\end{aligned}
$$

and

$$
\begin{aligned}
& \left\langle\left\langle\mu_{+}(x, t) \mu_{-}(0,0)\right\rangle\right\rangle_{\beta}^{\omega}=-e^{\left(\mathcal{E}[\omega]-\mathcal{E}\left[\frac{1}{2}+\omega\right]\right) x} \\
& \quad \times \sum_{\substack{k=0 \\
k \text { odd }}}^{\infty} \sum_{\epsilon_{1}, \ldots, \epsilon_{k}= \pm} \int_{\left\{\operatorname{Im}\left(\theta_{j}\right)=\epsilon_{j} 0^{+}\right\}} \frac{d \theta_{1} \cdots d \theta_{k} e^{\sum_{j=1}^{k} \epsilon_{j}\left(i m x \sinh \theta_{j}-i m t \cosh \theta_{j}\right)}}{k ! \prod_{j=1}^{k}\left(1+e^{2 \pi i \omega} e^{-\epsilon_{j} m \beta \cosh \theta_{j}}\right)}
\end{aligned}
$$




$$
\times i^{k} \prod_{j=1}^{k}\left({ }^{\omega} F_{\epsilon_{j}}^{\mu_{+}}\left(\theta_{j} ; L\right)\right)^{2} \prod_{1 \leq i<j \leq k} \tanh \left(\frac{\theta_{j}-\theta_{i}}{2}\right)^{2 \epsilon_{i} \epsilon_{j}}
$$

where $\omega=0$ or $\omega=\frac{1}{2}$, and we recall that $\mathcal{E}[0]=\mathcal{E}_{\mathrm{R}}$ and $\mathcal{E}[1 / 2]=\mathcal{E}_{\mathrm{NS}}$ are given in (7.7). In order to fully clarify the meaning of these finite-temperature correlation functions, we recall also that at imaginary time $t=i \mathrm{x}$ and at positive $x=\tau$, they correspond to the following correlation functions in the quantization on the circle:

$$
\begin{aligned}
& \left\langle\left\langle\sigma_{+}(\tau, i \mathrm{x}) \sigma_{-}(0,0)\right\rangle\right\rangle_{\beta}^{\omega}={ }_{\beta}\left\langle\operatorname{vac}_{\omega}|\sigma(\mathrm{x}, \tau) \sigma(0,0)| \operatorname{vac}_{\omega}\right\rangle_{\beta}, \\
& \left\langle\left\langle\mu_{+}(\tau, i \mathrm{x}) \mu_{-}(0,0)\right\rangle\right\rangle_{\beta}^{\omega}={ }_{\beta}\left\langle\operatorname{vac}_{\omega}|\mu(\mathrm{x}, \tau) \mu(0,0)| \operatorname{vac}_{\omega}\right\rangle_{\beta},
\end{aligned}
$$

where the vacuum is in the $\mathrm{R}$ sector if $\omega=0$, and in the NS sector if $\omega=1 / 2$.

Following [2], where techniques from [46, 47] were borrowed, Fredholm determinant representations can now easily be obtained for two-point functions from the formulas

$$
\operatorname{det}_{i, j}\left\{\frac{u_{i}-u_{j}}{u_{i}+u_{j}}\right\}= \begin{cases}\prod_{1 \leq i<j \leq k}\left(\frac{u_{i}-u_{j}}{u_{i}+u_{j}}\right)^{2} & k \text { even } \\ 0 & k \text { odd }\end{cases}
$$

and

$$
\operatorname{det}_{i, j}\left\{\frac{1}{u_{i}+u_{j}}\right\}=\frac{1}{2^{k} u_{1} \cdots u_{k}} \prod_{1 \leq i<j \leq k}\left(\frac{u_{i}-u_{j}}{u_{i}+u_{j}}\right)^{2} .
$$

Formula (7.21) gives

$$
\left\langle\left\langle\sigma_{+}(x, t) \sigma_{-}(0,0)\right\rangle\right\rangle_{\beta}^{\omega}=\operatorname{det}(\mathbf{1}+\mathbf{K}),
$$

where $\mathbf{K}$ is an integral operator with an additional index structure, defined by its action $(\mathbf{K} f)_{\epsilon}(\theta)=\sum_{\epsilon^{\prime}= \pm} \int_{-\infty}^{\infty} d \theta^{\prime} K_{\epsilon, \epsilon^{\prime}}\left(\theta, \theta^{\prime}\right) f_{\epsilon^{\prime}}\left(\theta^{\prime}\right)$ and its kernel

$$
K_{\epsilon, \epsilon^{\prime}}\left(\theta, \theta^{\prime}\right)=i\left({ }^{\omega} F_{\epsilon}^{\mu+}(\theta ; \beta)\right)^{2} \tanh \left(\frac{\theta^{\prime}-\theta}{2}\right)^{\epsilon \epsilon^{\prime}} \frac{e^{\epsilon(i m x \sinh \theta-i m t \cosh \theta)}}{1+e^{2 \pi i \omega} e^{-\epsilon m \beta \cosh \theta}} .
$$

Finally, in order to obtain two-point functions of disorder fields, we must consider the linear combinations $\sigma \pm \mu$. Formula (7.22) gives

$$
\left\langle\left\langle\left(\sigma_{+}(x, t)+\eta \mu_{+}(x, t)\right)\left(\sigma_{-}(0,0)+\eta \mu_{-}(0,0)\right)\right\rangle\right\rangle_{\beta}^{\omega}=\operatorname{det}\left(\mathbf{1}+\mathbf{J}^{(\eta)}\right)
$$

with $\eta= \pm$ and by definition $\left(\mathbf{J}^{(\eta)} f\right)_{\epsilon}(u)=\sum_{\epsilon^{\prime}= \pm} \int_{0}^{\infty} d u^{\prime} J_{\epsilon, \epsilon^{\prime}}^{(\eta)}\left(u, u^{\prime}\right) f_{\epsilon^{\prime}}\left(u^{\prime}\right)$ where the kernel is given by

$$
J_{\epsilon, \epsilon^{\prime}}^{(\eta)}\left(u, u^{\prime}\right)=-2 \eta i\left({ }^{\omega} F_{\epsilon}^{\mu}(\ln (u) ; \beta)\right)^{2} \frac{1}{\epsilon u+\epsilon^{\prime} u^{\prime}} \frac{e^{\frac{\epsilon}{2}\left(i m x\left(u-u^{-1}\right)-i m t\left(u+u^{-1}\right)\right)}}{1+e^{2 \pi i \omega} e^{-\frac{\epsilon m \beta}{2}\left(u+u^{-1}\right)}} .
$$

The interest in Fredholm determinant representations is, in part, that they can be used to efficiently obtain asymptotics of correlation functions.

Finally, we mention that these two-point functions in the Majorana theory can be used to evaluate the off-critical scaling limit of two-point functions in the quantum Ising chain (see, 
for instance, the book [48]). The quantum Ising chain is a quantum mechanical model with Hamiltonian

$$
H_{\text {Ising }}=-\sum_{j}\left(J s_{j}^{z} s_{j+1}^{z}+h s_{j}^{x}\right)
$$

with $J>0$. The spin variables $s_{j}^{x}$ and $s_{j}^{z}$ are in the spin-1/2 representation of $S U(2)$, and are two of the usual Pauli matrices on the $j^{\text {th }}$ two-dimensional space, the third one being $s_{j}^{y}$ :

$$
s^{x}=\left(\begin{array}{cc}
0 & 1 \\
1 & 0
\end{array}\right), \quad s^{y}=\left(\begin{array}{cc}
0 & -i \\
i & 0
\end{array}\right), \quad s^{z}=\left(\begin{array}{cc}
1 & 0 \\
0 & -1
\end{array}\right) .
$$

It is the "Hamiltonian limit" of the two-dimensional Ising classical statistical model. There is a value $h=h_{c}$ of the transverse magnetic field at which this model is critical. The conformal field theory that describes it is the free massless Majorana theory. For $h<h_{c}$, the system is ordered, and at zero temperature the average of $s_{j}^{z}$ is non-zero. On the other hand, for $h>h_{c}$, the system is disordered. As $h$ is made to approach $h_{c}$, the correlation length $\xi$ associated to the two-point function $\left\langle s_{j}^{z} s_{0}^{z}\right\rangle$ diverges. The scaling limit is obtained by looking at the situation where $h \rightarrow h_{c}$, while the inverse temperature is made to diverge as $J \beta \propto \xi$, and the distances between points in correlation functions are made to diverge as $|j| \propto \xi$. The quantum field theory model that describes the appropriately normalised correlation functions obtained in this limit is the free massive Majorana theory, the product of mass times position being equal to $m x=|j| / \xi$. If $h$ is sent to $h_{c}$ from below (ordered regime), then we have the correspondence

$$
Z^{-1} \xi^{\frac{1}{4}} \frac{\operatorname{Tr}\left(e^{-\beta H_{\text {Ising }}} s_{j}^{z}(t) s_{0}^{z}(0)\right)}{\operatorname{Tr}\left(e^{-\beta H_{\text {Ising }}}\right)} \rightarrow m^{-\frac{1}{4}}\left\langle\left\langle\sigma_{+}(x, t) \sigma_{-}(0,0)\right\rangle\right\rangle_{\beta}^{\frac{1}{2}},
$$

where

$$
s_{j}^{z}(t)=e^{-i t H_{\text {Ising }}} s_{j}^{z} e^{i t H_{\text {Ising }}}
$$

and $Z$ is a non-zero, non-universal number. On the other hand, if $h$ is sent to $h_{c}$ from above (disordered regime), then

$$
Z^{-1} \xi^{\frac{1}{4}} \frac{\operatorname{Tr}\left(e^{-\beta H_{\text {Ising }}} s_{j}^{z}(t) s_{0}^{z}(0)\right)}{\operatorname{Tr}\left(e^{-\beta H_{\text {Ising }}}\right)} \rightarrow m^{-\frac{1}{4}}\left\langle\left\langle\mu_{+}(x, t) \mu_{-}(0,0)\right\rangle\right\rangle_{\beta}^{\frac{1}{2}} .
$$

It is important to realise that the spin variables $s^{z}$ does not converge, in the scaling limit, to the twist fields $\sigma, \mu$; indeed, only its products converge to products of twist fields. This is clear, since the finite-temperature average of single twist fields are non-zero (but have non-trivial space dependence, as explained in Subsection 3.3), but finite-temperature averages of spin variables are zero (since at finite temperature, there can be no symmetry breaking). One should recall that the passage from the quantum Ising model to the Majorana theory involves writing the spin variables as exponentials of sums of (bilinear of) fermionic variables lying on a segment of the chain, and the two end-points of the segment correspond to two spin variables.

\section{Perspectives}

We have developed partly the concept of finite-temperature form factor in the general context of factorised scattering theory, and we completed the program in the case of the Majorana theory. The most important next step is, of course, to complete this program in models with non-trivial 
scattering. We believe that ideas concerning the relation between finite-temperature form factors and matrix elements in the quantization on the circle will lead to restrictive conditions that will greatly help fully fix finite-temperature form factors in interacting models. Also, the operator implementing the generalisation of CFT's "mapping to the cylinder" may be useful, and this method is not far from the explicit construction of "boundary-creating operator" in integrable boundary QFT. The generalisation to interacting models is a very important step, and will open the way to results about large-distance and large-time behaviours of correlation functions in interacting, integrable models.

Another interesting avenue is to generalise the program to the free Dirac theory; this should not pose any difficulties, and will clarify the structure of finite-temperature form factors of more general twist fields (two-point functions at finite-temperature were already studied in [49]). Then, it would be interesting to understand the structure for descendants of twist fields in such free fermionic models, perhaps using the operator $\Omega$ defined in (6.7) that provides a "mapping to the cylinder".

Finally, one would like to obtain the full large-time expansion of correlation functions in the quantum Ising model. Besides directly using the finite-temperature form factor expansion, it is possible that the Fredholm determinant representations obtained here can be used fruitfully for this purpose (work is in progress [31]).

\section{A OPE's in the Majorana theory}

The order and disorder fields have operator representations $\sigma_{ \pm}$and $\mu_{ \pm}$on $\mathcal{H}$. These operators are completely characterised by the leading terms in their OPEs with the fermion fields:

$$
\begin{aligned}
& \psi(x, t) \sigma_{+}(0, t) \sim \frac{1}{2 \sqrt{-\pi x-i 0^{+}}} \mu_{+}(0, t), \quad \sigma_{+}(0, t) \psi(x, t) \sim \frac{1}{2 \sqrt{-\pi x+i 0^{+}}} \mu_{+}(0, t), \\
& \psi(x, t) \sigma_{-}(0, t) \sim \frac{i}{2 \sqrt{\pi x+i 0^{+}}} \mu_{-}(0, t), \quad \sigma_{-}(0, t) \psi(x, t) \sim \frac{i}{2 \sqrt{\pi x-i 0^{+}}} \mu_{-}(0, t), \\
& \psi(x, t) \mu_{+}(0, t) \sim \frac{-i}{2 \sqrt{-\pi x-i 0^{+}}} \sigma_{+}(0, t), \quad \mu_{+}(0, t) \psi(x, t) \sim \frac{i}{2 \sqrt{-\pi x+i 0^{+}}} \sigma_{+}(0, t), \\
& \psi(x, t) \mu_{-}(0, t) \sim \frac{1}{2 \sqrt{\pi x+i 0^{+}}} \sigma_{-}(0, t), \quad \mu_{-}(0, t) \psi(x, t) \sim \frac{-1}{2 \sqrt{\pi x-i 0^{+}}} \sigma_{-}(0, t)
\end{aligned}
$$

and

$$
\begin{aligned}
\bar{\psi}(x, t) \sigma_{+}(0, t) & \sim \frac{1}{2 \sqrt{-\pi x+i 0^{+}}} \mu_{+}(0, t), & & \sigma_{+}(0, t) \bar{\psi}(x, t) \sim \frac{1}{2 \sqrt{-\pi x-i 0^{+}}} \mu_{+}(0, t), \\
\bar{\psi}(x, t) \sigma_{-}(0, t) & \sim-\frac{i}{2 \sqrt{\pi x-i 0^{+}}} \mu_{-}(0, t), & & \sigma_{-}(0, t) \bar{\psi}(x, t) \sim-\frac{i}{2 \sqrt{\pi x+i 0^{+}}} \mu_{-}(0, t), \\
\bar{\psi}(x, t) \mu_{+}(0, t) & \sim \frac{i}{2 \sqrt{-\pi x+i 0^{+}}} \sigma_{+}(0, t), & & \mu_{+}(0, t) \bar{\psi}(x, t) \sim \frac{-i}{2 \sqrt{-\pi x-i 0^{+}}} \sigma_{+}(0, t), \\
\bar{\psi}(x, t) \mu_{-}(0, t) & \sim \frac{1}{2 \sqrt{\pi x-i 0^{+}}} \sigma_{-}(0, t), & \mu_{-}(0, t) \bar{\psi}(x, t) & \sim \frac{-1}{2 \sqrt{\pi x+i 0^{+}}} \sigma_{-}(0, t),
\end{aligned}
$$

where everywhere, the square root is on its principal branch.

\section{Acknowledgments}

I am grateful to F. Essler for many useful discussions and continued interest in this work, and to A. Gamsa for reading through the manuscript. I would like to acknowledge support from an EPSRC (UK) post-doctoral fellowship (grant GR/S91086/01). 


\section{References}

[1] Kapusta J.I., Finite temperature field theory, Cambridge University Press, Cambridge, 1989.

[2] Doyon B., Finite-temperature form factors in the free Majorana theory, J. Stat. Mech. Theory Exp. (2005), P11006, 45 pages, hep-th/0506105.

[3] Bourbonnais C., Jerome D., The normal phase of quasi-one-dimensional organic superconductors, in Advances in Synthetic Metals, Twenty Years of Progress in Science and Technology, Editors P. Bernier, S. Lefrant and E. Bidan, Elsevier, New York, 1999.

[4] Gruner G., Density waves in solids, Addison-Wesley, Reading (MA), 1994.

[5] Essler F.H.L., Konik R.M., Applications of massive integrable quantum field theories to problems in condensed matter physics, in From Fields to Strings: Circumnavigating Theoretical Physics, Editors M. Shifman, A. Vainshtein and J. Wheater, Ian Kogan Memorial Collection, World Scientific, 2004.

[6] Vergeles S.N., Gryanik V.M., Two-dimensional quantum field theories having exact solutions, Yad. Fiz. 23 (1976), 1324-1334 (in Russian).

[7] Weisz P., Exact quantum sine-Gordon soliton form factors, Phys. Lett. B 67 (1977), 179-182.

[8] Karowski M., Weisz P., Exact form factors in (1+1)-dimensional field theoretic models with soliton behaviour, Nuclear Phys. B 139 (1978), 455-476.

[9] Berg B., Karowski M., Weisz P., Construction of Green's functions from an exact S-matrix, Phys. Rev. D 19 (1979), 2477-2479.

[10] Smirnov F.A., Form factors in completely integrable models of quantum field theory, World Scientific, Singapore, 1992.

[11] Zamolodchikov Al.B., Two-point correlation function in scaling Lee-Yang model, Nuclear Phys. B 348 (1991), 619-641.

[12] Matsubara T.M., A new approach to quantum-statistical mechanics, Progr. Theoret. Phys. 14 (1955), 351378.

[13] Kubo R., Statistical-mechanical theory of irreversible processes. I. General theory and simple applications to magnetic and conduction problems, J. Phys. Soc. Japan 12 (1957), 570-586.

[14] Martin C., Schwinger J., Theory of many-particle systems. I, Phys. Rev. 115 (1959), 1342-1373.

[15] Smirnov F.A., Quasi-classical study of form factors in finite volume, hep-th/9802132.

[16] Smirnov F.A., Structure of matrix elements in quantum Toda chain, hep-th/9805011.

[17] van Elburg R.A.J., Schoutens K., Form factors for quasi-particles in $c=1$ conformal field theory, J. Phys. A: Math. Gen. 33 (2000), 7987-8012, cond-mat/0007226.

[18] Mussardo G., Riva V., Sotkov G., Finite-volume form factors in semiclassical approximation, Nuclear Phys. B 670 (2003), 464-478, hep-th/0307125.

[19] Bugrij A.I., The correlation function in two dimensional Ising model on the finite size lattice. I, hep-th/0011104.

[20] Bugrij A.I., Form factor representation of the correlation function of the two dimensional Ising model on a cylinder, hep-th/0107117.

[21] Fonseca P., Zamolodchikov A.B., Ising field theory in a magnetic field: analytic properties of the free energy, J. Statist. Phys. 110 (2003), 527-590, hep-th/0112167.

[22] Leplae L., Umezawa H., Mancini F., Derivation and application of the boson method in superconductivity, Phys. Rep. 10 (1974), 151-272.

[23] Arimitsu T., Umezawa H., Non-equilibrium thermo field dynamics, Prog. Theoret. Phys. 77 (1987), 32-52.

[24] Arimitsu T., Umezawa H., General structure of non-equilibrium thermo field dynamics, Progr. Theoret. Phys. 77 (1987), 53-67.

[25] Henning P.A., Thermo field dynamics for quantum fields with continuous mass spectrum, Phys. Rep. 253 (1995), 235-381, nucl-th/9311001.

[26] Amaral R.L.P.G., Belvedere L.V., Two-dimensional thermofield bosonization, hep-th/0504012.

[27] Altshuler B.L., Konik R., Tsvelik A.M., Low temperature correlation functions in integrable models: derivation of the large distance and time asymptotics from the form factor expansion, Nuclear Phys. B 739 (2006), 311-327, cond-mat/0508618. 
[28] Sachdev S., The universal, finite temperature, crossover functions of the quantum transition in the Ising chain in a transverse field, Nuclear Phys. B 464 (1996), 576-595, cond-mat/9509147.

[29] Sachdev S., Young A.P., Low temperature relaxational dynamics of the Ising chain in a transverse field, Phys. Rev. Lett. 78 (1997), 2220-2223, cond-mat/9609185.

[30] Korepin V.E., Bogoliubov N.M., Izergin A.G., Quantum inverse scattering method and correlation functions, Cambridge University Press, Cambridge, 1993.

[31] Doyon B., Gamsa A., Work in progress.

[32] Balog J., Field theoretical derivation of the TBA integral equations, Nuclear Phys. B 419 (1994), 480-512.

[33] Leclair A., Mussardo G., Finite temperature correlation functions in integrable QFT, Nuclear Phys. B 552 (1999), 624-642, hep-th/9902075.

[34] Lukyanov S., Finite-temperature expectation values of local fields in the sinh-Gordon model, Nuclear Phys. B 612 (2001), 391-412, hep-th/0005027.

[35] Essler F., Konik R., Private communication.

[36] Wu T.T., McCoy B.M., Tracy C.A., Barouch E., Spin-spin correlation functions for the two-dimensional Ising model: exact theory in the scaling region, Phys. Rev. B 13 (1976), 316-374.

[37] Perk J.H.H., Equations of motion for the transverse correlations of the one-dimensional $X Y$-model at finite temperature, Phys. Lett. A $\mathbf{7 9}$ (1980), 1-2.

[38] Lisovyy O., Nonlinear differential equations for the correlation functions of the 2D Ising model on the cylinder, Adv. Theor. Math. Phys. 5 (2002), 909-922.

[39] Fonseca P., Zamolodchikov A.B., Ward identities and integrable differential equations in the Ising field theory, hep-th/0309228.

[40] Kadanoff L.P., Ceva H., Determination of an operator algebra for the two-dimensional Ising model, Phys. Rev. B 3 (1971), 3918-3939.

[41] Schroer B., Truong T.T., The order/disorder quantum field operators associated with the two-dimensional Ising model in the continuum limit, Nuclear Phys. B 144 (1978), 80-122.

[42] Doyon B., Lectures on integrable quantum field theory, http://www-thphys.physics.ox.ac.uk/user/BenjaminDoyon/lectures.pdf.

[43] van Hove L., Quantum field theory at positive temperature, Phys. Rep. 137 (1986), 11-20.

[44] Doyon B., Two-point functions of scaling fields in the Dirac theory on the Poincaré disk, Nuclear Phys. B 675 (2003), 607-630, hep-th/0304190.

[45] McCoy B.M., Wu T.T., The two-dimensional Ising model, Harvard University Press, Cambridge (MA), 1973.

[46] Babelon D., Bernard D., From form factors to correlation functions: the Ising model, Phys. Lett. B 288 (1992), 113-120.

[47] Leclair A., Lesage F., Sachdev S., Saleur H., Finite temperature correlations in the one-dimensional quantum Ising model, Nuclear Phys. B 482 (1996), 579-612, cond-mat/9606104.

[48] Itzykson C., Drouffe J.-M., Statistical field theory, Cambridge University Press, Cambridge, 1989.

[49] Lisovyy O., Tau functions for the Dirac operator on the cylinder, Comm. Math. Phys. 255 (2005), 61-95, hep-th/0312277. 